\title{
BROWNIAN MOTION WITH POLAR DRIFT
}

\author{
BY \\ R. J. WILLIAMS ${ }^{1}$
}

\begin{abstract}
Consider a strong Markov process $X^{0}$ that has continuous sample paths in $R^{d}(d \geqslant 2)$ and the following two properties.

(1) Away from the origin $X^{0}$ behaves like Brownian motion with a polar drift given in spherical polar coordinates by $\mu(\theta) / 2 r$. Here $\mu$ is a bounded Borel measurable function on the unit sphere in $R^{d}$, with average value $\bar{\mu}$.

(2) $X^{0}$ is absorbed at the origin.

It is shown that $X^{0}$ reaches the origin with probability zero or one as $\bar{\mu} \geqslant 2-d$ or $<2-d$. Indeed, $X^{0}$ is transient to $+\infty$ if $\bar{\mu}>2-d$ and null recurrent if $\bar{\mu}=2-d$. Furthermore, if $\bar{\mu}<2-d$ (i.e., $X^{0}$ reaches the origin), then $X^{0}$ does not approach the origin in any particular direction. Indeed, there is a single Martin boundary point for $X^{0}$ at the origin. The question of the existence and uniqueness of a strong Markov process with continuous sample paths in $R^{d}$ that behaves like $X^{0}$ away from the origin, but spends zero time there (in the sense of Lebesgue measure), is also resolved here.
\end{abstract}

1. Introduction. Let $X^{0}$ be a strong Markov process with continuous sample paths in $R^{d}(d \geqslant 2)$ such that the following two properties hold.

(i) In $R^{d} \backslash\{0\}, X^{0}$ behaves like a diffusion process with (local) generator

$$
L \equiv \frac{1}{2} \Delta+(2 r)^{-1} \mu(\theta) \frac{\partial}{\partial r} .
$$

(ii) $X^{0}$ is absorbed at the origin.

Here $\Delta$ is the usual Laplace operator in $R^{d}, \mu$ is a bounded Borel measurable function on the unit sphere $S^{d-1}$ in $R^{d}$, and $(r, \theta) \in(0, \infty) \times S^{d-1}$ denote polar coordinates in $R^{d} \backslash\{0\}$. Paraphrasing (i) we say that $X^{0}$ behaves like Brownian motion with polar drift $\mu(\theta) / 2 r$ in $R^{d} \backslash\{0\}$. The following questions are addressed in this paper. Does $X^{0}$ reach the origin (i.e. the pole)? If so, does it have an asymptotic direction of approach there? What is the nature of the Martin boundary for $X^{0}$ at the origin? Does there exist a strong Markov process with continuous sample paths in $R^{d}$ that behaves like $X^{0}$ away from the origin, but spends zero time there (in the sense of Lebesgue measure)?

There are two basic motivations for the study of this particular process $X^{0}$. The first is the desire to increase the detailed knowledge of the behavior of multidimensional diffusions near singular points of the coefficients. The second is the fact that $X^{0}$ is a basic "model" for comparison with processes having more general drift

Received by the editors, November 21, 1984.

1980 Mathematics Subject Classification. Primary 60J60, 60J65; Secondary 35J15.

Key words and phrases. Brownian motion, pole, drift, diffusion, Martin boundary, martingale, twisted product.

${ }^{1}$ Research supported in part by NSF Grant DMS 8319562. 
and/or diffusion coefficients, but for which direct computations are usually not possible.

This paper is divided into five sections. In $\S 2$, an alternative representation is given for $X^{0}$ as a "twisted product". This is similar to the skew product constructions of Itô and McKean [6, §7.15-\$7.17], which cover the special case $\mu \equiv$ constant. The difference here is that the radial part of the process can depend in a nontrivial way on the spherical part, via $\mu(\theta)$. The twisted product representation provides intuition for the results obtained in $\S 3$ and $\S 4$ on the behavior of $X^{0}$ near the origin. In $\S 3$ it is shown that starting from $x \neq 0, X^{0}$ hits the origin with probability zero or one, respectively, as

$$
\bar{\mu} \equiv \int_{S^{d-1}} \mu(\theta) d \bar{\sigma}(\theta)
$$

is $\geqslant(2-d)$ or $<(2-d)$. Here $\bar{\sigma}$ denotes surface area on $S^{d-1}$, normalized so that $\bar{\sigma}\left(S^{d-1}\right)=1$. Furthermore, $X^{0}$ is transient to $+\infty$ if $\bar{\mu}>2-d$ and null recurrent if $\bar{\mu}=2-d$. In $\S 4$, the asymptotic behavior of the resolvent of $X^{0}$ near the origin is obtained. This reflects the properties that $X^{0}$ does not approach the origin in any particular direction and that the Martin boundary is a single point there. The question of whether $X^{0}$ can be modified to a continuous strong Markov process that spends zero time at the origin is answered in $\$ 5$.

For the special case $\mu \equiv$ constant, the results obtained here are consistent with those that can be deduced by one-dimensional analysis using the skew product constructions of Itô and McKean [6]. The interested reader might like to compare this paper with that of Kendall [7] on "Pole seeking Brownian motion and bird navigation". The drift part of the two-dimensional diffusion studied there includes a singular term which has components in the radial and tangential directions (see [7, p. 381]).

For each domain $D \subset R^{d}$ and $n \geqslant 0, C^{n}(D)$ denotes the set of functions $f$ : $D \rightarrow R$ that are $n$-times continuously differentiable in $D$. The set of functions in $C^{n}(D)$ whose partial derivatives up to and including those of order $n$ are bounded on $D$ is denoted by $C_{b}^{n}(D)$. The symbol $C_{c}^{n}(D)$ denotes the set of functions in $C^{n}(D)$ that have compact support in $D$. If $n=0$, the superscript $n$ is usually omitted. The same notations will be used with $R^{1} \times S^{d-1}$ in place of $R^{d}$. The abbreviation "a.e." is used for "almost everywhere" with respect to Lebesgue measure on $R^{d}$.

The Laplace operator on $R^{d} \backslash\{0\}$ is given in polar coordinates by

$$
\Delta=\frac{\partial^{2}}{\partial r^{2}}+\frac{d-1}{r} \frac{\partial}{\partial r}+\frac{1}{r^{2}} \Delta_{S^{d}+1}
$$

where $\Delta_{S^{d-1}}$ denotes the Laplace-Beltrami operator on $S^{d-1}$. The set $S^{d-1}$ is endowed with the topology (and hence Borel $\sigma$-field) induced from $R^{d}$ and $\sigma$ denotes the surface measure there. The normalized surface measure $\sigma / \sigma\left(S^{d-1}\right)$ is denoted by $\bar{\sigma}$.

2. Twisted product representation. Given $\mu$ as described in $\S 1$, the existence and uniqueness (in law) of the process $X^{0}$ follows by applying the theory of Stroock and 
Varadhan $[13,14]$ to the diffusion with generator $L$ that is absorbed at the boundary of $R^{d} \backslash\{x:|x| \leqslant \varepsilon\}$, and then letting $\varepsilon \downarrow 0$. Indeed, $X^{0}$ is characterized (in law) as the unique process that has continuous paths in $R^{d}$ and associated probability measures $\left\{P_{x}^{0}\right\}$ (one for each starting point $x \in R^{d}$ ) satisfying the following three properties.

(i) $P_{x}^{0}\left(X^{0}(0)=x\right)=1$.

(ii) For each $f \in C_{c}^{2}\left(R^{d}\right)$ with compact support in $R^{d} \backslash\{0\}$,

$$
\left\{f\left(X^{0}\left(t \wedge \tau_{0}\right)\right)-\int_{0}^{t \wedge \tau_{0}}(L f)\left(X^{0}(s)\right) d s, \mathscr{F}_{t \wedge \tau_{0}}^{0}, t \geqslant 0\right\}
$$

is a $P_{x}^{0}$-martingale, where

$$
\mathscr{F}_{t}^{0}=\sigma\left\{X^{0}(s): 0 \leqslant s \leqslant t\right\} \quad \text { and } \quad \tau_{0}=\inf \left\{t \geqslant 0: X^{0}(t)=0\right\} .
$$

(iii) $P_{x}^{0}\left(X^{0}(t)=0\right.$ for all $\left.t \geqslant \tau_{0}\right)=1$.

In this section we give a representation for $X^{0}$ which provides useful intuition for the results given in later sections on the behavior of $X^{0}$ near the origin.

Suppose $(\Omega, \mathscr{G})$ is a fixed measurable space on which is defined a standard one-dimensional Brownian motion $B$, and an independent spherical Brownian motion $Z$ with paths in $S^{d-1}$. For each $t \in[0, \infty)$, define

$$
Y(t)=B_{1}(t)+\frac{1}{2}(d-2) t+\frac{1}{2} \int_{0}^{t} \mu(Z(s)) d s .
$$

Then $(Y, Z)$ is a diffusion process with continuous paths in $R^{1} \times S^{d-1}$ whose generator acting on functions in $C_{c}^{2}\left(R^{1} \times S^{d-1}\right)$ is given by

$$
L_{Y Z}=\frac{1}{2}\left(\frac{\partial^{2}}{\partial y^{2}}+(d-2) \frac{\partial}{\partial y}+\mu(z) \frac{\partial}{\partial y}+\Delta_{S^{d-1}}\right)
$$

for $(y, z) \in R^{1} \times S^{d-1}$. In particular, for each $g \in C_{c}^{2}\left(R^{1} \times S^{d-1}\right)$,

$$
\left\{g(Y(t), Z(t))-\int_{0}^{t}\left(L_{Y Z} g\right)(Y(s), Z(s)) d s, \mathscr{G}_{t}, t \geqslant 0\right\}
$$

is a $Q_{y, z}$-martingale, where $\left\{\mathscr{G}_{t}=\sigma\{(Y(s), Z(s)): 0 \leqslant s \leqslant t\}, t \geqslant 0\right\}$ is the natural filtration generated by $(Y, Z)$, and $Q_{y, z}$ is the probability measure on $(\Omega, \mathscr{G})$ associated with $(Y, Z)$ starting from $(y, z)$.

Next define

$$
A(t)=\int_{0}^{t} e^{2 Y(s)} d s \quad \text { for all } t \geqslant 0
$$

and

$$
A_{\infty}=\int_{0}^{\infty} e^{2 Y(s)} d s
$$

Let $A^{-1}$ denote the (continuous) functional inverse of $A$, with $A^{-1}(t) \equiv \infty$ if $t \geqslant A_{\infty}$. For each $t \in\left[0, A_{\infty}\right)$, define $X^{*}(t)$ in polar coordinates by

$$
X^{*}(t)=(R(t), \Theta(t))
$$

where

$$
R(t)=e^{Y\left(A^{-1}(t)\right)} \quad \text { and } \quad \Theta(t)=Z\left(A^{-1}(t)\right)
$$


Further define

$$
X^{*}(t)=0 \in R^{d} \text { for all } t \geqslant A_{\infty} .
$$

Then we have the following

THEOREM 2.1. $X^{*}$ is a representation for $X^{0}$, i.e., $X^{*}$ is equivalent in law to $X^{0}$.

Proof. By construction, $X^{*}$ has continuous paths in $R^{d}$ and is absorbed at the origin. Thus, it suffices to verify that property (ii) of the characterization of $X^{0}$ holds for $X^{*}$, for each starting point $x \in R^{d}$. For this it is enough to verify that, for each $x \in R^{d} \backslash\{0\}, 0<\varepsilon<1$ and $f \in C_{c}^{2}\left(R^{d} \backslash\{0\}\right)$,

$$
\left\{f\left(X^{*}\left(t \wedge \tau_{\varepsilon}^{*}\right)\right)-\int_{0}^{t \wedge \tau_{\varepsilon}^{*}}(L f)\left(X^{*}(s)\right) d s, \mathscr{F}_{t \wedge \tau_{*}^{*}}^{*}, t \geqslant 0\right\}
$$

is a $Q_{y, z}$-martingale, where $(y, z)=(\ln |x|, x /|x|) \in R^{1} \times S^{d-1}$,

$$
\tau_{\varepsilon}^{*}=\inf \left\{s \geqslant 0:\left|X^{*}(s)\right| \leqslant \varepsilon \text { or }\left|X^{*}(s)\right| \geqslant \varepsilon^{-1}\right\},
$$

and $\mathscr{F}_{t}{ }^{*}=\sigma\left\{X^{*}(s): 0 \leqslant s \leqslant t\right\}$. Given $0<\varepsilon<1$ and $f(r, \theta) \in C_{c}^{2}\left(R^{d} \backslash\{0\}\right)$, let

$$
\tau_{\varepsilon}^{Y}=\inf \left\{s \geqslant 0: Y(s) \leqslant \ln \varepsilon \text { or } Y(s) \geqslant \ln \left(\varepsilon^{-1}\right)\right\}
$$

and define $g \in C_{c}^{2}\left(R^{1} \times S^{d-1}\right)$ by

$$
g(y, z)=f\left(e^{y}, z\right) \quad \text { for all }(y, z) \in R^{1} \times S^{d-1} .
$$

Then by the martingale property $(2.4)$ of $(Y, Z)$ and Doob's stopping theorem, since $E^{Q_{v: z}}\left(\tau_{\varepsilon}^{Y}\right)<\infty$, we have

$$
\begin{aligned}
\left\{g\left(Y\left(A^{-1}(t) \wedge \tau_{\varepsilon}^{Y}\right), Z\left(A^{-1}(t) \wedge \tau_{\varepsilon}^{Y}\right)\right)\right. & \\
& -\int_{0}^{A^{-1}(t) \wedge \tau_{\varepsilon}^{Y}}\left(L_{Y Z} g\right)(Y(s), Z(s)) d s, \mathscr{G}_{A^{-1}(t) \wedge \tau_{\varepsilon}^{Y}, t \geqslant 0} \geqslant
\end{aligned}
$$

is a $Q_{y, z}$-martingale for each $(y, z) \in R^{1} \times S^{d-1}$. Now, by (2.3),

$$
\left(L_{Y Z} g\right)(y, z)=\left\{\frac{1}{2}\left(e^{2 y} \frac{\partial^{2}}{\partial r^{2}}+(d-1+\mu(z)) e^{y} \frac{\partial}{\partial r}+\Delta_{S^{d}}\right) f\right\}\left(e^{y}, z\right) .
$$

Also, $A^{-1}(t) \wedge \tau_{\varepsilon}^{Y}=A^{-1}\left(t \wedge \tau_{\varepsilon}^{*}\right)$. By substituting the above in (2.11) and changing the variable of integration there to $u=A(s)$ (so that $d u=e^{2 Y(s)} d s$ ), we obtain

$$
\begin{aligned}
\left\{f\left(\exp \left(Y\left(A^{-1}\left(t \wedge \tau_{\varepsilon}^{*}\right)\right)\right), Z\left(A^{-1}\left(t \wedge \tau_{\varepsilon}^{*}\right)\right)\right)\right. \\
\left.-\int_{0}^{t \wedge \tau_{\varepsilon}^{*}}(L f)\left(\exp \left(Y\left(A^{-1}(u)\right)\right), Z\left(A^{-1}(u)\right)\right) d u, \mathscr{G}_{A^{-1}\left(t \wedge \tau_{\varepsilon}^{*}\right)}, t \geqslant 0\right\}
\end{aligned}
$$

is a $Q_{y, z}$-martingale. Here (cf. (1.1)-(1.2)),

$$
L=\frac{1}{2}\left(\frac{\partial^{2}}{\partial r^{2}}+r^{-1}(d-1+\mu(\theta)) \frac{\partial}{\partial r}+r^{-2} \Delta_{S^{d-1}}\right) .
$$

Recalling the definition (2.7)-(2.8) of $X^{*}$ and noting that $\mathscr{F}_{t \wedge \tau_{f}^{*}}^{*} \subset \mathscr{G}_{A^{-1}\left(t \wedge \tau_{\varepsilon}^{*}\right)}$, we see that the desired martingale property $(2.10)$ holds.

Henceforth we shall use $X^{*}$ as our representation for $X^{0}$.

By dividing (2.2) by $t$ and letting $t \rightarrow \infty$, we obtain, for $(y, z) \in R^{1} \times S^{d-1}$

$$
\lim _{t \rightarrow \infty} \frac{Y(t)}{t}=\frac{1}{2}\left\{d-2+\int_{S^{d-1}} \mu(\theta) d \bar{\sigma}(\theta)\right\} \quad Q_{y, z} \text {-a.s. }
$$


since the steady-state distribution for $Z$ on $S^{d-1}$ is uniform. Thus, if

$$
\bar{\mu} \equiv \int_{S^{d-1}} \mu(\theta) d \bar{\sigma}(\theta)<2-d,
$$

then $Q_{y, z}$-a.s. there is a constant $c>0$ and time $t_{0}$ (depending on $\omega \in \Omega$ ) such that $Y(t) \leqslant-c t$ for all $t \geqslant t_{0}$. This implies $A_{\infty}=\int_{0}^{\infty} e^{2 Y(s)} d s<\infty Q_{y, z}$-a.s. and, consequently (cf. (2.9)), $\tau_{0} \equiv \inf \left\{t \geqslant 0: X^{0}(t)=0\right\}<\infty P_{x}^{0}$-a.s. for all $x \in R^{d}$. On the other hand, if $\bar{\mu}>2-d$, it follows that $A_{\infty}=\infty Q_{y, z}$-a.s., and for $x \neq 0, P_{x}^{0}$-a.s., $\tau_{0}=\infty$ and $\left|X^{0}(t)\right|=R(t)=e^{Y\left(A^{-1}(t)\right)} \rightarrow \infty$ as $t \rightarrow \infty$. In the next section these results will be strengthened to show that if $x \neq 0$ and $\bar{\mu}=2-d$, then $P_{x}^{0}$-a.s., $\tau_{0}=\infty$. However, $X^{0}$ is (finely) null recurrent then.

In case $\bar{\mu}<2-d, A^{-1}(t) \uparrow \infty$ as $t \uparrow \tau_{0}<\infty P_{x}^{0}$-a.s. $(x \neq 0)$. Since $\Theta(t)=$ $Z\left(A^{-1}(t)\right)$ and $Z$ is positive recurrent on $S^{d-1}$, it then follows that $X^{0}$ does not have an asymptotic direction of approach to the origin. This property is reflected in the asymptotic behavior obtained in $\S 4$ for the resolvent of $X^{0}$ near the origin.

3. Is the pole reached? To determine whether the origin (the pole of $\mu(\theta) / 2 r$ ) is reached by the process $X^{0}$, we seek a nonconstant function $u$ such that $u\left(X^{0}(\cdot)\right)$ is a local martingale under $P_{x}^{0}$ for each $x \neq 0$. For this, since $L$ is the generator of $X^{0}$, we seek a function $u$ such that (at least in a weak sense)

$$
L u=0 \quad \text { in } R^{d} \backslash\{0\}
$$

and $u$ has either a minimum (of zero) or a singularity at the origin. Because $L$ is homogeneous in $r$, it is natural to look for a $u$ of the following form for some $\alpha \in R^{1}$ :

$$
u(r, \theta)= \begin{cases}r^{\alpha} \phi_{\alpha}(\theta) & \text { if } \alpha \in R^{1} \backslash\{0\} \\ \ln r+\chi(\theta) & \text { if } \alpha=0\end{cases}
$$

where $\phi_{\alpha}>0$ on $S^{d-1}$.

Since $\mu$ was only assumed to be bounded and measurable, in general the most regularity we can expect for $\phi_{\alpha}$ (or $\chi$ ) is that it lie in some Sobolev space contained in $C^{1}\left(S^{d-1}\right)$ such that (3.1) holds in the sense of (Schwartz) distributions. Let $p$ be a fixed real number such that $p>d-1$, and let $W^{2, p}\left(S^{d-1}\right)$ denote the Sobolev space of functions on $S^{d-1}$ that have derivatives through order 2 in $L^{p}\left(S^{d-1}\right)$. By the Sobolev embedding theorem (cf. [5, §7.7]), $W^{2, p}\left(S^{d-1}\right) \subset C^{1}\left(S^{d-1}\right)$. It is shown below that there is an $\alpha$ (depending on $\mu$ and $d$ ) and an associated $\phi_{\alpha}>0$ (or $\chi$ if $\alpha=0)$ in $W^{2, p}\left(S^{d-1}\right)$ such that $u$ given by (3.2) satisfies (3.1) in the sense of distributions. An extended version of the martingale property (2.1) is then applied to $u$ to show that, starting from $x \neq 0, X^{0}$ reaches the origin with $P_{x}^{0}$-probability zero or one as $\alpha \leqslant 0$ or $\alpha>0$. Although a closed form expression is not known for $\alpha$ (except when $\mu \equiv$ constant), the following criterion is given for determining the sign of $\alpha: \alpha<0,=0$, or $>0$ as

$$
\mu \equiv \int_{S^{d},} \mu(\theta) d \bar{\sigma}(\theta)
$$

is $>2-d,=2-d$, or $<2-d$ (note the reversal of the inequality signs here). 
To prove the above, we first determine whether there is a $u$ of the form (3.2) with $\alpha \neq 0$. By the definition (2.12) of $L$, there is such a $u$ if and only if there is $\alpha \neq 0$ and $\phi_{\alpha} \in W^{2, p}\left(S^{d-1}\right)$ satisfying $\phi_{\alpha}>0$ on $S^{d-1}$ and

$$
\Delta_{S^{d-1}} \phi_{\alpha}+\{\alpha \mu+\alpha(\alpha+d-2)\} \phi_{\alpha}=0 \text { on } S^{d-1} .
$$

Here and henceforth, an equation involving functions in Sobolev spaces is interpreted in the weak sense of distributions. If $\mu \equiv$ constant $\neq 2-d$, then $\alpha=2-d-\mu$ and $\phi_{\alpha} \equiv 1$ determine a suitable $u$. But in general, $\alpha$ and $\phi_{\alpha}$ cannot be determined explicitly. This prompts the following restatement $(\mathrm{Q})$ of the question of the existence of $\alpha$ and $\phi_{\alpha}$, in terms of an eigenvalue problem.

For each $\alpha \in R^{1}$, for all $\lambda \in R^{1}$ sufficiently negative, $\left(-\Delta_{S^{d-1}}-\alpha \mu-\lambda\right)^{-1}$ is a bounded positive linear operator from $L^{p}\left(S^{d-1}\right)$ to $W^{2, p}\left(S^{d-1}\right)$. Since $W^{2, p}\left(S^{d-1}\right)$ is compactly embedded in $L^{p}\left(S^{d-1}\right)$, it then follows by the Krein-Rutman [8, Theorem 6.3] theory of positive linear operators that, for each $\alpha \in R^{1}$, there is a smallest eigenvalue $\lambda(\alpha)$ and associated (strictly) positive eigenfunction $\psi_{\alpha} \in$ $W^{2, p}\left(S^{d-1}\right)$ satisfying $\int_{S^{d-1}} \psi_{\alpha}^{2} d \bar{\sigma}=1$ and

$$
\Delta_{S^{d-1}} \psi_{\alpha}+\{\alpha \mu+\lambda(\alpha)\} \psi_{\alpha}=0 \text { on } S^{d-1} .
$$

The question of the existence of $\alpha \neq 0$ and $\phi_{\alpha}>0$ satisfying (3.4) can then be rephrased as follows.

$$
\text { Is there an } \alpha \neq 0 \text { such that } \lambda(\alpha)=\alpha(\alpha+d-2) \text { ? }
$$

To answer this, the following properties of $\lambda(\alpha)$ and $\psi_{\alpha}$ are used (cf. [5]). For $\alpha=0, \lambda(0)=0$ and $\psi_{0}=1 /\left(\bar{\sigma}\left(S^{d-1}\right)\right)^{1 / 2}$. For each $\alpha \in R^{1}$,

$$
\lambda(\alpha)=\inf _{0 \neq \psi \in W^{2, p}\left(S^{d-1}\right)}\left\{\int_{S^{d-1}} \psi\left(-\Delta_{S^{d-1}} \psi-\alpha \mu \psi\right) d \bar{\sigma} / \int_{S^{d-1}} \psi^{2} d \bar{\sigma}\right\} .
$$

It follows that $\lambda(\alpha)$ is a concave function of $\alpha$, and by setting $\psi \equiv 1$ in (3.6) we see that $\lambda(\alpha) \leqslant|\alpha|\|\mu\|_{\infty}$ where $\|\mu\|_{\infty}=\sup \left\{|\mu|(\theta): \theta \in S^{d-1}\right\}$. By the perturbation theory of $\left(\Delta_{S^{\alpha-1}}+\alpha \mu+\lambda\right)^{-1}$ (cf. [3, Lemma 1.3]), $\alpha \rightarrow \lambda(\alpha) \in R^{1}$ and $\alpha \rightarrow \psi_{\alpha} \in$ $W^{2, p}\left(S^{d-1}\right)$ are real analytic functions of $\alpha$. By integrating (3.5) over $S^{d-1}$ and using Green's identity: $\int_{S^{d-1}} \Delta_{S^{d-1}} \psi_{\alpha} d \bar{\sigma}=0$, we conclude that

$$
\lambda(\alpha)=-\left\{\alpha \int_{S^{d-1}} \mu \psi_{\alpha} d \bar{\sigma}\right\} /\left\{\int_{S^{d-1}} \psi_{\alpha} d \bar{\sigma}\right\} .
$$

Then, by differentiating (3.7) with respect to $\alpha$ and setting $\alpha=0$, we obtain

$$
\lambda^{\prime}(0)=-\int_{S^{d-1}} \mu(\theta) d \bar{\sigma}(\theta) \equiv-\bar{\mu} .
$$

Thus, $\eta(\alpha) \equiv \lambda(\alpha)-\alpha(\alpha+d-2)$ is a strictly concave function of $\alpha, \eta(0)=0$ and $\eta(\alpha) \rightarrow-\infty$ as $\alpha \rightarrow \pm \infty$. It follows that there is (a unique) $\alpha>0$ (resp. $\alpha<0$ ) such that $\lambda(\alpha)=\alpha\left(\alpha+d-2\right.$ ) if and only if $\eta^{\prime}(0)>0$ (resp. $\left.\eta^{\prime}(0)<0\right)$. By combining this with (3.8) and giving a separate argument for the exceptional case $\eta^{\prime}(0)=0$, we obtain the following. 
THEOREM 3.1. If $\bar{\mu}<2-d$ (resp. $>2-d$ ), there is (a unique) $\alpha>0$ (resp. $\alpha<0)$ such that (3.5) holds with $\lambda(\alpha)=\alpha(\alpha+d-2)$. Then by setting $\phi_{\alpha}=\psi_{\alpha}$ for this $\alpha$, we obtain a solution $u(r, \theta)=r^{\alpha} \phi_{\alpha}(\theta)$ of (3.1) with $\phi_{\alpha} \in W^{2, p}\left(S^{d-1}\right) \subset$ $C^{1}\left(S^{d-1}\right)$ and $\phi_{\alpha}>0$ on $S^{d-1}$. If $\bar{\mu}=2-d$, there is $\chi \in W^{2, p}\left(S^{d-1}\right) \subset C^{1}\left(S^{d-1}\right)$ such that

$$
u(r, \theta)=\ln r+\chi(\theta)
$$

is a solution of (3.1).

Proof. The cases $\bar{\mu}<2-d$ and $\bar{\mu}>2-d$ follow from the discussion above and the observation that $\eta^{\prime}(0)$ is $>0$ (resp. $\left.<0\right)$ as $\bar{\mu} \equiv-\lambda^{\prime}(0)$ is $<2-d$ (resp. $>2-d$ ).

So suppose $\bar{\mu}=2-d$. By the definition (2.12) of $L$, there is a $u$ of the form (3.9) satisfying (3.1) if and only if there is $\chi \in W^{2, p}\left(S^{d-1}\right)$ satisfying

$$
\Delta_{S^{d-1}} \chi=-(d-2+\mu) \text { on } S^{d-1} \text {. }
$$

Now $\lambda=0 \in R^{1}$ is the smallest eigenvalue of $\left(\Delta_{S^{d-1}}+\lambda\right)^{-1}$ and the constant function is the solution of the corresponding homogeneous adjoint problem. It then follows by the Fredholm alternative (cf. $[5, \$ 5.4]$ ) that there is a solution $\chi \in$ $W^{2, p}\left(S^{d-1}\right)$ satisfying (3.10) if and only if

$$
\int_{S^{d-1}}(d-2+\mu) d \bar{\sigma}=0 .
$$

But (3.11) is precisely the condition that $\bar{\mu}=2-d$.

Henceforth, $\alpha$ will be used to denote the distinguished real number such that there is a solution $u$ of the form (3.2) satisfying (3.1). In particular, $\alpha=0$ if $\bar{\mu}=2-d$.

The function $u$ will now be used to determine whether the origin is reached by $X^{0}$. But first we must show that $X^{0}$ escapes from any bounded, punctured neighborhood of the origin in finite (expected) time. For this it is expeditious to use the times at which $X^{0}$ first reaches a level set of the function $v$ defined on $R^{d} \backslash\{0\}$ by

$$
v= \begin{cases}u & \text { if } \alpha>0 \\ e^{u} & \text { if } \alpha=0 \\ 1 / u & \text { if } \alpha<0\end{cases}
$$

and defined at the origin by $v(0)=0$. The function $v$ is continuous on $R^{d}$ and $v(r, \theta)$ increases with $r \in(0, \infty)$ for each fixed $\theta \in S^{d-1}$. For each $r \geqslant 0$, define

$$
\tau_{r}=\inf \left\{t \geqslant 0: v\left(X^{0}(t)\right)=r\right\},
$$

and for $0 \leqslant r<K<\infty$, let $\tau_{r K}=\tau_{r} \wedge \tau_{K}$.

Lemma 3.1. Let $x \in R^{d} \backslash\{0\}$. Then for any $K \geqslant v(x)$, we have

$$
E^{P_{x}^{0}}\left[\int_{0}^{\tau_{0} K} h\left(X^{0}(s)\right) d s\right] \leqslant g(u(x)),
$$

where for $(r, \theta) \in R^{d} \backslash\{0\}$,

$$
h(r, \theta)= \begin{cases}\phi_{\alpha}^{(2 / \alpha)-2}(\theta)\left(\alpha^{2} \phi_{\alpha}^{2}+\left|\nabla_{S^{d-1}} \phi_{\alpha}\right|^{2}\right)(\theta) & \text { if } \alpha \neq 0 \\ e^{2 \chi(\theta)}\left(1+\left|\nabla_{S^{d-1}} \chi\right|^{2}\right)(\theta) & \text { if } \alpha=0\end{cases}
$$


and if $\alpha \in(0, \infty) \backslash\{2\}$,

$$
g(y)=\left\{\begin{array}{l}
0 \quad \text { for } y=0, \\
\alpha^{2} y\left(K^{(2 / \alpha)-1}-y^{(2 / \alpha)-1}\right) /(2-\alpha) \quad \text { for } 0 \leqslant y \leqslant K, \\
\alpha K^{2 / \alpha}(1-y / K) \quad \text { for } y \geqslant K,
\end{array}\right.
$$

if $\alpha=2$,

$$
g(y)=\left\{\begin{array}{l}
0 \quad \text { for } y=0, \\
2 y(\ln K-\ln y) \quad \text { for } 0<y \leqslant K \\
2 K(1-(y / K)) \quad \text { for } y \geqslant K
\end{array}\right.
$$

if $\alpha=0$,

$$
g(y)=\left\{\begin{array}{l}
\left(K^{2}-e^{2 y}\right) / 2 \quad \text { for }-\infty<y<\ln K, \\
K^{2}(\ln K-y) \quad \text { for } y \geqslant \ln K,
\end{array}\right.
$$

if $\alpha<0$,

$$
g(y)= \begin{cases}-2 \alpha K^{1-(2 / \alpha)}\left(y-K^{-1}\right) /(2-\alpha) & \text { for } 0 \leqslant y<K^{-1}, \\ \alpha^{2}\left(K^{-2 / \alpha}-y^{2 / \alpha}\right) /(2-\alpha) & \text { for } y \geqslant K^{-1}\end{cases}
$$

Remarks. (1) Since $\phi_{\alpha}$ (or $\chi$ if $\alpha=0$ ) is in $C^{1}\left(S^{d-1}\right)$ and $\phi_{\alpha}>0$, it follows that $h \in C\left(R^{d} \backslash\{0\}\right)$ and there are constants $c_{1}, c_{2}$ such that

$$
0<c_{1} \leqslant h \leqslant c_{2} \text { on } R^{d} \backslash\{0\} .
$$

(2) The relation (3.14) actually holds with equality in place of inequality there. This is proved in Theorem 3.3, since it requires the result of Theorem 3.2, which in turn depends on the above lemma.

Proof. On $R^{d} \backslash\{0\}$, define $f=g(u)$. Then $f \in C^{1}\left(R^{d} \backslash\{0\}\right) \cap W_{\mathrm{loc}}^{2, p}\left(R^{d} \backslash\{0\}\right)$. Here $W_{\text {loc }}^{2, p}\left(R^{d} \backslash\{0\}\right)$ denotes the space of functions that are in the Sobolev space $W^{2, p}(D)$ for each domain $D \subset R^{d} \backslash\{0\}$ such that $\bar{D}$ is a compact subset of $R^{d} \backslash\{0\}$. Now,

$$
L f=\frac{1}{2} g^{\prime \prime}(u)|\nabla u|^{2}+g^{\prime}(u) L u=\frac{1}{2} g^{\prime \prime}(u)|\nabla u|^{2},
$$

where

$$
|\nabla u|^{2}=\left\{\begin{array}{l}
r^{2 \alpha-2}\left(\alpha^{2} \phi_{\alpha}^{2}+\left|\nabla_{S^{d-1}} \phi_{\alpha}\right|^{2}\right)=u^{2-(2 / \alpha)} h \quad \text { if } \alpha \neq 0, \\
r^{-2}\left(1+\left|\nabla_{S^{d-1}} \chi\right|^{2}\right)=e^{-2 u} h \quad \text { if } \alpha=0 .
\end{array}\right.
$$

The function $g$ was chosen such that, if $\alpha>0$,

$$
g^{\prime \prime}(y)= \begin{cases}-2 y^{(2 / \alpha)-2} & \text { for } 0<y<K, \\ 0 & \text { for } y>K\end{cases}
$$

if $\alpha=0$,

$$
g^{\prime \prime}(y)= \begin{cases}-2 e^{2 y} & \text { for }-\infty<y<\ln K, \\ 0 & \text { for } y>\ln K,\end{cases}
$$


and if $\alpha<0$,

$$
g^{\prime \prime}(y)= \begin{cases}0 & \text { for } 0<y<K^{-1}, \\ -2 y^{(2 / \alpha)-2} & \text { for } y>K^{-1} .\end{cases}
$$

By combining these with (3.21)-(3.22), we see that

$$
L f(z)= \begin{cases}-h(z) & \text { for } 0<v(z)<K, \\ 0 & \text { for } v(z)>K .\end{cases}
$$

It is readily verified that $f$ is nonnegative and bounded on $\left\{z \in R^{d}: 0<v(z)<K\right\}$, that $f(z)=0$ on $v^{-1}(K)$ and $\lim _{z \rightarrow 0} f(z)=0$ if $\alpha>0$. Consistent with the last property, we define $f(0)=0$ if $\alpha>0$.

For $0<r<v(x)$, let $D_{r K}=\left\{z \in R^{d}: r<v(z)<K\right\}$. It follows by the same kind of approximation argument as in Stroock and Varadhan [12, II, Theorem 11.2] that the martingale property (2.1) extends to $f \in C^{1}\left(R^{d} \backslash\{0\}\right) \cap W_{\text {loc }}^{2, p}\left(R^{d} \backslash\{0\}\right)$ with $\tau_{r K}$ in place of $\tau_{0}$. Hence, by (3.23), for all $t \geqslant 0$ :

$$
E^{P_{x}^{0}}\left[f\left(X^{0}\left(t \wedge \tau_{r K}\right)\right)\right]+E^{P_{x}^{0}}\left[\int_{0}^{t \wedge \tau_{r K}} h\left(X^{0}(s)\right) d s\right]=f(x) .
$$

By letting $t \rightarrow \infty$ and then $r \rightarrow 0$ in the above, and using the nonnegativity of $f$ and Fatou's lemma, we obtain

$$
E^{P_{x}^{0}}\left[\int_{0}^{\tau_{0} K} h\left(X^{0}(s)\right) d s\right] \leqslant f(x) .
$$

Since $f(x)=g(u(x))$, the proof is complete.

Corollary 3.1. For each $x \in R^{d} \backslash\{0\}$ and $K \geqslant v(x)$,

$$
E^{P_{x}^{0}}\left[\tau_{0 K}\right]<\infty \text {. }
$$

Proof. Since $h \geqslant c_{1}>0$ on $R^{d} \backslash\{0\}$, this follows immediately from (3.14).

TheOREM 3.2. Let $x \in R^{d} \backslash\{0\}$. Then,

$$
P_{x}^{0}\left(\tau_{0}<\infty\right)= \begin{cases}1 & \text { if } \alpha>0 \\ 0 & \text { if } \alpha \leqslant 0\end{cases}
$$

where $\alpha>0$ or $\alpha \leqslant 0$ as $\bar{\mu}<2-d$ or $\bar{\mu} \geqslant 2-d$.

Proof. Let $0<\varepsilon<v(x)<K<\infty$ and recall that

$$
\tau_{\varepsilon K}=\inf \left\{t \geqslant 0: v\left(X^{0}(t)\right)=\varepsilon \text { or } v\left(X^{0}(t)\right)=K\right\} .
$$

As in the proof of Lemma 3.1 above, the martingale property (2.1), with $\tau_{\varepsilon K}$ in place of $\tau_{0}$, can be extended to

$$
u \in C^{1}\left(R^{d} \backslash\{0\}\right) \cap W_{\mathrm{loc}}^{2, p}\left(R^{d} \backslash\{0\}\right) .
$$

Thus $\left\{u\left(X^{0}\left(t \wedge \tau_{\varepsilon K}\right)\right), \mathscr{F}_{t \wedge \tau_{\varepsilon K}}^{0}, t \geqslant 0\right\}$ is a $P_{x}^{0}$-martingale. Since $u$ is bounded on $\bar{D}_{\varepsilon K}=\{z: \varepsilon \leqslant v(z) \leqslant K\}$ and $P_{x}^{0}\left(\tau_{\varepsilon K}<\infty\right)=1$, by (3.26), after taking the expected value of $u\left(X^{0}\left(t \wedge \tau_{\varepsilon K}\right)\right)$ and letting $t \rightarrow \infty$, we conclude that

$$
E^{P_{x}^{0}}\left[u\left(X^{0}\left(\tau_{\varepsilon K}\right)\right)\right]=u(x) .
$$


If $\alpha>0$,

$$
u\left(X^{0}\left(\tau_{\varepsilon K}\right)\right)=\varepsilon l_{\left\{\tau_{\varepsilon}<\tau_{K}\right\}}+K 1_{\left\{\tau_{K}<\tau_{\epsilon}\right\}} \quad P_{x}^{0} \text {-a.s. }
$$

By combining this with (3.28) we obtain

$$
P_{x}^{0}\left(\tau_{\varepsilon}<\tau_{K}\right)=(K-u(x)) /(K-\varepsilon) .
$$

Since $\tau_{0 K}<\infty P_{x}^{0}$-a.s. by (3.26), we can let $\varepsilon \downarrow 0$ in the above to conclude that

$$
P_{x}^{0}\left(\tau_{0}<\tau_{K}\right)=1-\{u(x) / K\},
$$

and then let $K \rightarrow \infty$ to obtain $P_{x}^{0}\left(\tau_{0}<\infty\right)=1$ if $\alpha>0$. On the other hand, for $\alpha \leqslant 0$, by using the definitions of $\tau_{\varepsilon K}$ and $v$ (see (3.12)), we see that (3.28) implies

$$
P_{x}^{0}\left(\tau_{\varepsilon}<\tau_{K}\right)= \begin{cases}\left(u(x)-K^{-1}\right) /\left(\varepsilon^{-1}-K^{-1}\right) & \text { if } \alpha<0 \\ (\ln K-u(x)) /(\ln K-\ln \varepsilon) & \text { if } \alpha=0 .\end{cases}
$$

Then letting $\varepsilon \downarrow 0$ and using (3.26) we conclude that

$$
P_{x}^{0}\left(\tau_{0}<\tau_{K}\right)=0 \quad \text { if } \alpha \leqslant 0 .
$$

By letting $t \rightarrow \infty$, and then $r \rightarrow 0$ in (3.24), and using (3.27) to conclude that $f\left(X^{0}\left(\tau_{0 K}\right)\right)=0 P_{x}^{0}$-a.s., we obtain the following strengthened version of Lemma 3.1. To emphasize its importance for work in later sections, it is labelled as a theorem.

THeOREM 3.3. Let $x \in R^{d} \backslash\{0\}, K \geqslant v(x)$, and $g, h$ be defined as in Lemma 3.1. Then,

$$
E^{P_{x}^{0}}\left[\int_{0}^{\tau_{0 K}} h\left(X^{0}(s)\right) d s\right]=g(u(x)) .
$$

By using (3.30), the following result on transience/recurrence of $X^{0}$ can be proved in a similar manner to that employed in Williams [16]. For details and terminology, the interested reader is referred to that paper.

Corollary 3.2. If $\bar{\mu}>2-d$,

$$
P_{x}^{0}\left(\liminf _{t \rightarrow \infty}\left|X^{0}(t)\right|=+\infty\right)=1
$$

for all $x \in R^{d} \backslash\{0\}$. If $\bar{\mu}=2-d$, starting from $x \neq 0, X^{0}$ is (finely) null recurrent on $R^{d}$.

4. An ergodic property. For each $\gamma>0$ and $k \in C_{b}\left(R^{d}\right)$, let $R_{\gamma}^{0} k$ denote the stopped resolvent defined by

$$
R_{\gamma}^{0} k(x)=E^{P_{x}^{0}}\left[\int_{0}^{\tau_{0}} e^{-\gamma t} k\left(X^{0}(t)\right) d t\right] \text { for all } x \in R^{d} .
$$

The following result plays a key role in determining whether there is a strong Markov process with continuous paths in $R^{d}$ that behaves like $X^{0}$ in $R^{d} \backslash\{0\}$, but spends zero time at the origin. It also implies that there is a single point of the Martin boundary for $X^{0}$ at the origin (cf. Rogers [11, §4]). In the sequel, uniformly as $|x| \rightarrow 0$ means uniformly in the spherical coordinate $\theta \equiv x /|x|$. 
THEOREM 4.1. For each $\gamma>0, \beta>0$ and $k \in C_{b}\left(R^{d}\right)$ having compact support in $R^{d} \backslash\{0\}$,

$$
\frac{R_{\gamma}^{0} k(x)}{R_{\beta}^{0} 1(x)} \rightarrow \begin{cases}c(\gamma, \beta, k) & \text { if } \alpha<2, \\ 0 & \text { if } \alpha \geqslant 2\end{cases}
$$

uniformly as $|x| \rightarrow 0$ for $x \in R^{d} \backslash\{0\}$, where $c(\gamma, \beta, k)$ is a constant depending on $\gamma, \beta, k$ such that if $0 \equiv k \geqslant 0$, then $c(\gamma, \beta, k)>0$.

The proof of Theorem 4.1 relies on the following two theorems. Specifically, in Theorem 4.2 we establish the asymptotic behavior as $|x| \rightarrow 0$ of $R_{\gamma}^{0} k(x)$ for $k \in C_{b}\left(R^{d}\right)$ and $\alpha<2$, and vital estimates on $E^{P_{x}^{0}}\left[e^{-\lambda \tau_{0 e}} ; \tau_{\varepsilon}<\tau_{0}\right]$ and $E^{P_{x}^{0}}\left[e^{-\beta \tau_{0}}\right]$ follow from Theorem 4.3.

THEOREM 4.2. Suppose $\alpha<2$. For each $\gamma>0$ and $k \in C_{b}\left(R^{d}\right)$ there is a constant $c=c(\gamma, k)<\infty$ such that

$$
\begin{aligned}
R_{\gamma}^{0} k(x) / u(x) & \rightarrow c \quad \text { if } \alpha>0, \\
R_{\gamma}^{0} k(x) \rightarrow c & \text { if } \alpha \leqslant 0
\end{aligned}
$$

uniformly as $|x| \rightarrow 0$ for $x \in R^{d} \backslash\{0\}$.

The following lemmas are fundamental to the proof of Theorem 4.2. The first lemma follows immediately from Theorem 3.3 and (3.20). Here the relation $\sim$ is defined such that, for two real-valued functions $f$ and $g$ with the same domain, $f \sim g$ if and only if there are constants $a_{1}>0$ and $a_{2}>0$ such that $a_{1} f \leqslant g \leqslant a_{2} f$.

$$
E^{P_{x}^{0}}\left[\tau_{0 \varepsilon}\right] \sim \begin{cases}\alpha^{2} u(x)\left(\varepsilon^{(2 / \alpha)-1}-u(x)^{(2 / \alpha)-1}\right) /(2-\alpha) & \text { if } \alpha \in(0, \infty) \backslash\{2\}, \\ 2 u(x) \ln (\varepsilon / u(x)) & \text { if } \alpha=2, \\ \frac{1}{2}\left(\varepsilon^{2}-e^{2 u(x)}\right) & \text { if } \alpha=0, \\ \alpha^{2}\left(\varepsilon^{-(2 / \alpha)}-(u(x))^{2 / \alpha}\right) /(2-\alpha) & \text { if } \alpha<0 .\end{cases}
$$

The next lemma gives a Riesz-type decomposition for the stopped resolvent.

LEMMA 4.2. Let $x \in R^{d}$ and $\tau$ be a stopping time (with respect to $\left\{\mathscr{F}_{t}^{0}\right\}$ ) such that $\tau \leqslant \tau_{0} P_{x}^{0}$-a.s. and $E^{P_{x}^{0}}[\tau]<\infty$. Then for each $\gamma>0$ and $k \in C_{b}\left(R^{d}\right), f=R_{\gamma}^{0} k$ satisfies

$$
f(x)=E^{P_{x}^{0}}\left[\int_{0}^{\tau}\left\{k\left(X^{0}(t)\right)-\gamma f\left(X^{0}(t)\right)\right\} d t\right]+E^{P_{x}^{0}}\left[f\left(X^{0}(\tau)\right)\right] .
$$

Proof. The first term in the right member of (4.5) is finite because $f$ and $k$ are bounded and we assumed that $E^{P_{x}^{0}}[\tau]<\infty$. The proof of this lemma involves standard manipulations using the strong Markov property and Fubini's theorem (cf. Chung [2, p. 203]). The details are left to the reader.

The following "scaling" lemma derives from the homogeneity of $L$ with respect to the radial coordinate $r$. 
Lemma 4.3. For $\lambda>0$ and $x \in R^{d}, X^{0}(\cdot)$ under $P_{x}^{0}$ is equivalent in law to $\lambda^{-1} X^{0}\left(\lambda^{2} \cdot\right)$ under $P_{\lambda x}^{0}$.

REMARK. Here "equivalent in law" means that the probability measures induced on the space of continuous paths in $R^{d}$ by the two processes are the same.

Proof. By the characterization given for $X^{0}$ in $\S 2$, it suffices to verify that properties (i)-(iii) listed there hold with $\lambda^{-1} X^{0}\left(\lambda^{2} \cdot\right)$ and $P_{\lambda x}^{0}$, respectively, in place of $X^{0}(\cdot)$ and $P_{x}^{0}$, for each $x \in R^{d}$. Indeed, property (i) is readily verified and so too is (iii) with the aid of the identity

$$
\tau_{0}\left(X^{0}(\cdot)\right)=\lambda^{2} \tau_{0}\left(\lambda^{-1} X^{0}\left(\lambda^{2} \cdot\right)\right)
$$

where $\tau_{0}(w)=\inf \{t \geqslant 0: w(t)=0\}$, for $w=X^{0}(\cdot)$ or $\lambda^{-1} X^{0}\left(\lambda^{2} \cdot\right)$. To verify (ii), let $f \in C_{c}^{2}\left(R^{d}\right)$ with compact support in $R^{d} \backslash\{0\}$. By applying property (ii) of $P_{\lambda x}^{0}$ to $f\left(\lambda^{-1} \cdot\right)$ and using the homogeneity of $L$ with respect to $r$, we obtain

$$
\begin{aligned}
\left\{f \left(\lambda ^ { - 1 } X ^ { 0 } \left(\lambda^{2} t \wedge\right.\right.\right. & \left.\left.\tau_{0}\left(X^{0}\right)\right)\right) \\
& \left.-\int_{0}^{\lambda^{2} t \wedge \tau_{0}\left(X^{0}\right)} \lambda^{-2}(L f)\left(\lambda^{-1} X^{0}(s)\right) d s, \mathscr{F}_{\lambda^{2} t \wedge \tau_{0}\left(X^{0}\right)}, t \geqslant 0\right\}
\end{aligned}
$$

is a $P_{\lambda x}^{0}$-martingale. Then by changing the variable of time integration from $s$ to $\lambda^{-2} s$ and using (4.6), we conclude that

$$
\left\{f\left(\lambda^{-1} X^{0}\left(\lambda^{2}\left(t \wedge \tau_{0}^{\lambda}\right)\right)\right)-\int_{0}^{1 \wedge \tau_{0}^{\lambda}}(L f)\left(\lambda^{-1} X^{0}\left(\lambda^{2} s\right)\right) d s, \mathscr{F}_{t \wedge \tau_{0}^{\lambda}}^{\lambda}, t \geqslant 0\right\}
$$

is a $P_{\lambda x}^{0}$-martingale, where

$$
\tau_{0}^{\lambda} \equiv \tau_{0}\left(\lambda^{-1} X^{0}\left(\lambda^{2} \cdot\right)\right) \quad \text { and } \quad \mathscr{F}_{t}^{\lambda}=\sigma\left\{\lambda^{-1} X^{0}\left(\lambda^{2} s\right): 0 \leqslant s \leqslant t\right\} .
$$

As in $\S 2, R(t)$ and $\Theta(t)$ are used below to denote the radial and spherical parts, respectively, of $X^{0}(t)$ for all $t<\tau_{0}$.

Fix $\varepsilon>0$. For each $x=(r, \theta) \in v^{-1}\left(\frac{1}{2} \varepsilon\right)$ and each Borel measurable set $F \subset S^{d-1}$, let $\Pi(\theta, F)$ denote the conditional probability defined by

$$
\Pi(\theta, F)=\frac{P_{x}^{0}\left(\tau_{\varepsilon}<\tau_{0} ; \Theta\left(\tau_{\varepsilon}\right) \in F\right)}{P_{x}^{0}\left(\tau_{\varepsilon}<\tau_{0}\right)} .
$$

Here, by (3.27) and (3.29) we have

$$
P_{x}^{0}\left(\tau_{\varepsilon}<\tau_{0}\right)= \begin{cases}1 & \text { if } \alpha \leqslant 0 \\ \frac{1}{2} & \text { if } \alpha>0\end{cases}
$$

For each $x=(r, \theta)$ and $F, \Pi(\theta, \cdot)$ is a (Borel) probability measure on $S^{d-1}$ and $\Pi(\cdot, F)$ is Borel measurable on $S^{d-1}$. The latter follows from the continuity of $\Phi_{\varepsilon / 2}(\theta)=\left(r_{\varepsilon / 2}(\theta), \theta\right)$, where $r_{\varepsilon / 2}(\theta)$ is the unique value in $(0, \infty)$ such that $v\left(r_{\varepsilon / 2}(\theta), \theta\right)=\frac{1}{2} \varepsilon$, and the Borel measurability of $P_{x}^{0}\left(\tau_{\varepsilon}<\tau_{0} ; \Theta\left(\tau_{\varepsilon}\right) \in F\right)$ on $\{x: 0<v(x)<\varepsilon\}$ (cf. Chung [2, p. 75; p. 153, ex. 6]). Thus, $\Pi$ is the transition probability function for a Markov chain with state space $S^{d-1}$. For each integer $n \geqslant 1$, the $n$-step transition probabilities of this chain are given by

$$
\Pi^{n}(\xi, F)=\int_{S^{d-1}} \Pi\left(\xi, d \xi_{1}\right) \cdots \int_{S^{d-1}} \Pi\left(\xi_{n-2}, d \xi_{n-1}\right) \Pi\left(\xi_{n-1}, F\right)
$$

for any $\xi \in S^{d-1}$ and Borel set $F \subset S^{d-1}$. 
Next, an ergodic result is proved for this Markov chain. Following Revuz [10, p. 201], we say that $\left\{\Pi^{n}\right\}_{n=1}^{\infty}$ converges uniformly to a (Borel) probability measure $\kappa$ on $S^{d-1}$ if and only if

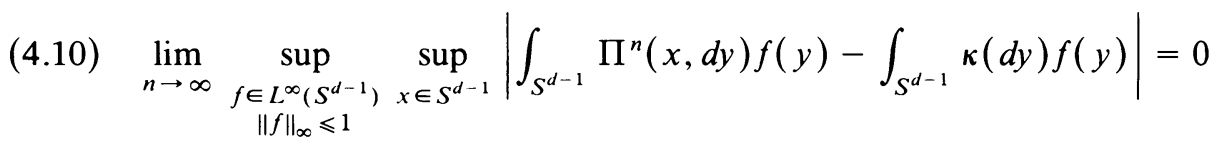

where $\|\cdot\|_{\infty}$ denotes the essential supremum norm on $L^{\infty}\left(S^{d-1}\right)$.

LEMMA 4.4. $\left\{\Pi^{n}\right\}_{n=1}^{\infty}$ converges uniformly to a (Borel) probability measure $\kappa$ on $S^{d-1}$.

Proof. It is shown below that there are constants $c>0$ and $\delta>0$, and a (Borel) probability measure $\nu$ on $S^{d-1}$ such that

$$
\Pi(\xi, \cdot) \geqslant c \nu(\cdot)^{\delta} \text { for all } \xi \in S^{d-1} .
$$

This implies that the Markov chain associated with $\Pi$ is an aperiodic Harris chain (see Revuz [10, pp. 92, 194-196]) and that it satisfies Doeblin's condition (see [10, p. 210]). The desired result then follows immediately from Revuz [10, pp. 203, 211].

For the verification of (4.11), by (4.7)-(4.8), it suffices to show that there are constants $c>0$ and $\delta>0$, and a (Borel) probability measure $\rho$ on $v^{-1}(\varepsilon)$ such that

$$
\inf _{x \in v^{-1}(\varepsilon / 2)} P_{x}^{0}\left(X^{0}\left(\tau_{\varepsilon} \wedge \tau_{\varepsilon / 4}\right) \in F\right) \geqslant c \rho(F)^{\delta}
$$

for all Borel sets $F \subset v^{-1}(\varepsilon)$. Indeed, since $\theta \rightarrow \Phi_{\varepsilon}(\theta)=\left(r_{\varepsilon}(\theta), \theta\right)$ is one-to-one and continuous, $\nu \equiv \rho\left(\Phi_{\varepsilon}(\cdot)\right)$ would then satisfy (4.11).

For each $x=(r, \theta) \in R^{d} \backslash\{0\}$, let $\vec{\mu}(x)=\mu(\theta) \vec{e}_{r}$ where $\vec{e}_{r}$ denotes the unit vector in the outward radial direction at $x$. Let $B_{0}$ be a $d$-dimensional Brownian motion starting from the origin, defined on a probability space $\left(\tilde{\Omega}, \tilde{\mathscr{F}}, \tilde{P}_{0}\right)$. For each $(\omega, \tilde{\omega}) \in \Omega \times \tilde{\Omega}$, let

$$
B(t ; \omega, \tilde{\omega})=\left\{\begin{array}{l}
X^{0}(t, \omega)-\int_{0}^{t} \frac{\vec{\mu}\left(X^{0}(s, \omega)\right)}{2\left|X^{0}(s, \omega)\right|} d s \quad \text { for } 0 \leqslant t \leqslant \tau(\omega), \\
B(\tau(\omega))+B_{0}(t-\tau(\omega) ; \tilde{\omega}) \text { for } t>\tau(\omega)
\end{array}\right.
$$

where $\tau(\omega) \equiv\left(\tau_{\varepsilon} \wedge \tau_{\varepsilon / 4}\right)(\omega)$. Then for each $x \in G_{\varepsilon} \equiv\left\{z \in R^{d}: \varepsilon / 4 \leqslant v(z) \leqslant \varepsilon\right\}$, on $\left(\bar{\Omega}, \overline{\mathscr{F}}, \bar{P}_{x}\right) \equiv\left(\Omega \times \tilde{\Omega}, \mathscr{F} \times \tilde{\mathscr{F}}, P_{x}^{0} \times \tilde{P}_{0}\right), B$ is a $d$-dimensional Brownian motion starting from $x$. This follows from the facts that under $P_{x}^{0}, X^{0}$ behaves in $R^{d} \backslash\{0\}$ like Brownian motion starting from $x$ with a polar drift of the form $\mu(\theta) / 2 r$, and $\tau(\omega)$ is independent of $B_{0}(\cdot ; \tilde{\omega})$. For all $(t, \omega, \tilde{\omega}) \in[0, \infty) \times \bar{\Omega}$, let

$$
\bar{X}(t ; \omega, \tilde{\omega})=B(t ; \omega, \tilde{\omega})+\int_{0}^{t \wedge \tau(\omega)} \frac{\vec{\mu}\left(X^{0}(s, \omega)\right)}{2\left|X^{0}(s, \omega)\right|} d s
$$

so that $\bar{X}(t ; \omega, \tilde{\omega})=X^{0}(t, \omega)$ for all $t \leqslant \tau(\omega)$. On $\bar{\Omega}$, define $\tau(\omega, \tilde{\omega})=\tau(\omega)$,

$$
\eta_{\varepsilon}=\inf \{t \geqslant 0: v(B(t)) \leqslant \varepsilon / 4 \text { or } \geqslant \varepsilon\},
$$


and

$$
\chi_{\varepsilon}=\exp \left(\int_{0}^{\eta_{\varepsilon}} \frac{\vec{\mu}(B(s))}{2|B(s)|} d B(s)-\frac{1}{8} \int_{0}^{\eta_{\varepsilon}} \frac{|\vec{\mu}(B(s))|^{2}}{|B(s)|^{2}} d s\right) .
$$

Now,

$$
m_{\varepsilon} \equiv \sup _{x \in G_{\varepsilon}}\{|\vec{\mu}(x)| / 2|x|\}<\infty, \quad P_{x}\left(\eta_{\varepsilon}<\infty\right)=1,
$$

and by (3.26), $P_{x}^{0}\left(\tau_{\varepsilon} \wedge \tau_{\varepsilon / 4}<\infty\right)=1$. It then follows from a version of Girsanov's change of measure formula (cf. Lipster and Shiryayev [9, Theorem 7.10]) that, for any Borel set $F \subset v^{-1}(\varepsilon)$ and $x \in G_{\varepsilon}$,

$$
P_{x}^{0}\left(X^{0}\left(\tau_{\varepsilon} \wedge \tau_{\varepsilon / 4}\right) \in F\right)=\bar{P}_{x}(\bar{X}(\tau) \in F)=E^{\bar{P}_{x}}\left[\chi_{\varepsilon} 1_{F}\left(B\left(\eta_{\varepsilon}\right)\right)\right] .
$$

Now by Chung [2, (X), p. 148], there is $a_{\varepsilon}>0$ such that, for all $0<a<a_{\varepsilon}$,

$$
\sup _{x \in G_{\varepsilon}} E^{\bar{P}_{x}}\left[\exp \left(a \eta_{\varepsilon}\right)\right]<\infty .
$$

Moreover, by the Cauchy-Schwarz inequality, for $b>0$ and $x \in G_{\varepsilon}$, (4.15)

$$
\begin{aligned}
&\left(E^{\bar{P}_{x}}\left[\chi_{\varepsilon}^{-b}\right]\right)^{2} \leqslant E^{\bar{P}_{x}}\left[\exp \left\{-\int_{0}^{\eta_{e}} \frac{b \vec{\mu}(B(s))}{|B(s)|} d B(s)-\frac{1}{2} \int_{0}^{\eta_{\varepsilon}}\left(\frac{b|\vec{\mu}(B(s))|}{|B(s)|^{2}}\right)^{2} d s\right\}\right] \\
& \cdot E^{\bar{P}_{x}}\left[\exp \left\{\int_{0}^{\eta_{e}} \frac{\left(2 b^{2}+b\right)|\vec{\mu}(B(s))|^{2}}{4|B(s)|} d s\right\}\right] .
\end{aligned}
$$

Since $E^{\bar{P}_{x}}\left[\chi_{\varepsilon}\right]=1$, with $-2 b \vec{\mu}$ in place of $\vec{\mu}$, the first factor of the product in (4.15) is identically one. By combining this with the definition of $m_{\varepsilon}$, we see that the right member of (4.15) is dominated above by

$$
E^{\bar{P}_{x}}\left[\exp \left\{\left(2 b^{2}+b\right) m_{\varepsilon}^{2} \eta_{\varepsilon}\right\}\right] .
$$

Then using (4.14) we conclude that there is $b_{\varepsilon}>0$ such that, for $0<b \leqslant b_{\varepsilon}$,

$$
\sup _{x \in G_{\varepsilon}} E^{\bar{P}_{x}}\left[\chi_{\varepsilon}^{-b}\right]<\infty .
$$

Choose real numbers $p_{\varepsilon}$ and $q_{\varepsilon}$ such that $1<q_{\varepsilon}<p_{\varepsilon}<\infty, 1 / p_{\varepsilon}+1 / q_{\varepsilon}=1$ and $q_{\varepsilon} / p_{\varepsilon} \leqslant b_{\varepsilon}$. Then by Hölder's inequality, for $x \in G_{\varepsilon}$ we have

$$
\begin{aligned}
E^{\bar{P}_{x}}\left[\chi_{\varepsilon} 1_{F}\left(B\left(\eta_{\varepsilon}\right)\right)\right] & \geqslant\left(E^{\bar{P}_{x}}\left[1_{F}\left(B\left(\eta_{\varepsilon}\right)\right)\right]\right)^{p_{\varepsilon}} /\left(E^{\bar{P}_{x}}\left[\chi_{\varepsilon}^{-q_{\varepsilon} / p_{\varepsilon}}\right]\right)^{p_{\varepsilon} / q_{\varepsilon}} \\
& \geqslant\left(E^{\bar{P}_{x}}\left[1_{F}\left(B\left(\eta_{\varepsilon}\right)\right)\right]\right)^{p_{\varepsilon}} / c_{1, \varepsilon}
\end{aligned}
$$

where

$$
c_{1, \varepsilon} \equiv \sup _{x \in G_{\varepsilon}}\left(E^{\bar{P}_{x}}\left[\chi_{\varepsilon}^{-q_{\varepsilon} / p_{\varepsilon}}\right]\right)^{p_{\varepsilon} / q_{\varepsilon}}<\infty
$$

by (4.16).

Recall (cf. (3.12)) that $v(r, \theta)=r^{\beta} \psi(\theta)$ where $\beta=|\alpha|$ if $\alpha \neq 0$ or 1 if $\alpha=0$, and $\psi(\theta)=\phi_{\alpha}(\theta)$ if $\alpha>0,\left(\phi_{\alpha}(\theta)\right)^{-1}$ if $\alpha<0$, or $e^{\chi(\theta)}$ if $\alpha=0$. Since $\psi$ is strictly positive and $C^{1}$ on $S^{d-1}$, it follows that, for any $a>0, v^{-1}(a)=\left\{\left(r_{a}(\theta), \theta\right)\right.$ : $\left.v\left(r_{a}(\theta), \theta\right)=a, \theta \in S^{d-1}\right\}$ is the graph of a $C^{1}$ function on $S^{d-1}$. Hence the boundary of the domain $\left\{x \in R^{d}: \varepsilon / 4<v(x)<\varepsilon\right\}$ is $C^{1}$. 
Now by the properties of harmonic measure for domains with $C^{1}$ (hence Lipschitz) boundary (see Dahlberg [4, p. 276]), and Harnack's inequality (see Gilbarg and Trudinger [5, Theorem 2.5]), it follows that there are constants $c_{2, \varepsilon}>0$ and $\delta_{\varepsilon}>0$ such that

$$
\inf _{x \in v^{-1}(\varepsilon / 2)} E^{\bar{P}_{x}}\left[1_{F}\left(B\left(\eta_{\varepsilon}\right)\right)\right] \geqslant c_{2, \varepsilon} \bar{\sigma}(F)^{\delta_{\varepsilon}}
$$

where $\bar{\sigma}$ denotes the surface measure on $v^{-1}(\varepsilon)$, normalized so that $\bar{\sigma}\left(v^{-1}(\varepsilon)\right)=1$. By combining this with (4.13) and (4.17), we conclude that (4.12) holds with $c=c_{2, \varepsilon}^{p_{\epsilon}} / c_{1, \varepsilon}$, $\delta=p_{\varepsilon} \delta_{\varepsilon}$ and $\rho=\bar{\sigma}$.

The following lemma establishes a link between the ergodic behavior of the Markov chain on $S^{d-1}$ (determined by $\Pi$ ) and the asymptotic behavior of $P_{x}^{0}$ as $|x| \rightarrow 0$.

LEMMA 4.5. For each integer $n \geqslant 1$, each $x_{n}=\left(r_{n}, \theta_{n}\right) \in v^{-1}\left(\varepsilon 2^{-n}\right)$ and each Borel set $F \subset S^{d-1}$, we have

$$
P_{x_{n}}^{0}\left(\tau_{\varepsilon}<\tau_{0} ; \Theta\left(\tau_{\varepsilon}\right) \in F\right)=\bar{\Pi}^{n}\left(\theta_{n}, F\right)
$$

where

$$
\bar{\Pi}^{n}= \begin{cases}2^{-n} \Pi^{n} & \text { if } \alpha>0, \\ \Pi^{n} & \text { if } \alpha \leqslant 0 .\end{cases}
$$

Proof. The proof is by induction on $n$. The result is true for $n=1$ by definition (see (4.7)-(4.8)). Suppose it is true for some $n \geqslant 1$. Then,

$$
\begin{aligned}
P_{x_{n+1}}^{0}\left(\tau_{\varepsilon}<\tau_{0} ;\right. & \left.\Theta\left(\tau_{\varepsilon}\right) \in F\right) \\
& =P_{x_{n+1}}^{0}\left(\tau_{\varepsilon^{-n}}<\tau_{0} ; P_{X^{0}\left(\tau_{\varepsilon 2^{-n}}\right)}^{0}\left(\tau_{\varepsilon}<\tau_{0} ; \Theta\left(\tau_{\varepsilon}\right) \in F\right)\right) \\
& =\int_{S^{d-1}} P_{x_{n+1}}^{0}\left(\tau_{\varepsilon 2^{-n}}<\tau_{0} ; \Theta\left(\tau_{\varepsilon 2^{-n}}\right) \in d \xi\right) \bar{\Pi}^{n}(\xi, F) .
\end{aligned}
$$

Here we have used the strong Markov property to obtain the second line and the induction hypothesis for the last line. Let $\lambda_{n}=2^{n /|\alpha|}$ if $\alpha \neq 0$ or $\lambda_{n}=2^{n}$ if $\alpha=0$. Then $\lambda_{n} x_{n+1} \in v^{-1}(\varepsilon / 2)$ and by the scaling lemma 4.3, the last line of (4.19) is equal to

$$
\int_{S^{d-1}} P_{\lambda_{n} x_{n+1}}^{0}\left(\tau_{\varepsilon}<\tau_{0} ; \Theta\left(\tau_{\varepsilon}\right) \in d \xi\right) \bar{\Pi}^{n}(\xi, F)=\int_{S^{d-1}} \bar{\Pi}\left(\theta_{n+1}, d \xi\right) \bar{\Pi}^{n}(\xi, F) .
$$

The result now follows for $n+1$ from the definitions of $\bar{\Pi}^{n+1}$ and $\Pi^{n+1}$.

In the following, $\kappa_{\varepsilon}$ is defined to be equal to $\kappa / \varepsilon$ if $\alpha>0$ or $\kappa$ if $\alpha \leqslant 0$, where $\kappa$ is the probability measure of Lemma 4.4, which may itself depend on $\varepsilon$.

LEMMA 4.6. For each continuous function $f$ on $S^{d-1}$ and for $q$ defined on $R^{d} \backslash\{0\}$ by

$$
q= \begin{cases}u & \text { if } \alpha>0 \\ 1 & \text { if } \alpha \leq 0\end{cases}
$$

we have

$$
\left\{\int_{S^{d-1}} P_{x}^{0}\left(\tau_{\varepsilon}<\tau_{0} ; \Theta\left(\tau_{\varepsilon}\right) \in d \xi\right) f(\xi) d \xi\right\} / q(x) \rightarrow \int_{S^{d-1}} \kappa_{\varepsilon}(d \xi) f(\xi)
$$

uniformly in $\theta$ as $x=(r, \theta) \in R^{d} \backslash\{0\}$ tends to 0 . 
Proof. Since $v(x) \sim|x|^{|\alpha|}$ if $\alpha \neq 0$ or $v(x) \sim|x|$ if $\alpha=0$, it suffices to prove that (4.20) holds as $v(x) \rightarrow 0$.

Consider $n \geqslant 1$ and $x \in R^{d} \backslash\{0\}$ such that $v(x)<\varepsilon 2^{-n}$. Then by Lemma 4.5, the left member of (4.20) is equal to

$$
\int_{S^{d-1}} P_{x}^{0}\left(\tau_{\varepsilon 2^{-n}}<\tau_{0} ; \Theta\left(\tau_{\varepsilon 2^{-n}}\right) \in d \xi\right) \int_{S^{d-1}} \bar{\Pi}^{n}\left(\xi, d \xi_{1}\right) f\left(\xi_{1}\right) / q(x) .
$$

By (3.27) and (3.29),

$$
P_{x}^{0}\left(\tau_{\varepsilon 2^{-n}}<\tau_{0}\right)= \begin{cases}2^{n} u(x) / \varepsilon & \text { if } \alpha>0, \\ 1 & \text { if } \alpha \leqslant 0 .\end{cases}
$$

Thus, $a_{n} P_{x}^{0}\left(\tau_{\varepsilon 2^{-n}}<\tau_{0} ; \Theta\left(\tau_{\varepsilon 2^{-n}}\right) \in d \xi\right) / q(x)$, where $a_{n}=\varepsilon 2^{-n}$ if $\alpha>0$ or $a_{n}=1$ if $\alpha \leqslant 0$, defines a (Borel) probability measure on $S^{d-1}$. By combining this with the definitions of $\bar{\Pi}^{n}$ and $\kappa_{\varepsilon}$, and the uniform convergence in Lemma 4.4, it follows that (4.21) converges to the right member of (4.20) as $n \rightarrow \infty$, i.e. as $v(x) \rightarrow 0$.

Proof of Theorem 4.2. Suppose $\alpha<2$ and $\gamma, k$ are defined as in the statement of the theorem. Then by the Feller continuity of $P_{x}^{0}$ on $R^{d} \backslash\{0\}$ (cf. Stroock and Varadhan $[13,14]), f(x)=R_{\gamma}^{0} k(x)$ is continuous there and $f_{\varepsilon}$ defined by

$$
f_{\varepsilon}(\theta)=f\left(r_{\varepsilon}(\theta), \theta\right) \text { for all } \theta \in S^{d-1}
$$

is continuous on $S^{d-1}$. Here $r_{\varepsilon}$ is defined in the same manner as $r_{\varepsilon / 2}$ (see the paragraph following (4.8)). Then for $q$ as defined in Lemma 4.6, we have

$$
\begin{aligned}
E^{P_{x}^{0}}\left[f\left(X^{0}\left(\tau_{0 \varepsilon}\right)\right)\right] / q(x) & =\left\{\int_{S^{d-1}} P_{x}^{0}\left(\tau_{\varepsilon}<\tau_{0} ; \Theta\left(\tau_{\varepsilon}\right) \in d \xi\right) f_{\varepsilon}(\xi)\right\} / q(x) \\
& \rightarrow \int_{S^{d-1}} \kappa_{\varepsilon}(d \xi) f_{\varepsilon}(\xi) \equiv c_{\varepsilon}
\end{aligned}
$$

uniformly as $|x| \rightarrow 0$ in $R^{d} \backslash\{0\}$.

By combining this with Lemmas 4.1 and 4.2 (setting $\tau=\tau_{0 \varepsilon}$ in the latter), since $\alpha<2$, we obtain

$$
\lim \left(\begin{array}{c}
\sup \\
\text { inf }
\end{array}\right)_{|x| \rightarrow 0} f(x) / q(x)=c_{\varepsilon}+O(\beta(\varepsilon))
$$

where

$$
\beta(\varepsilon)= \begin{cases}\varepsilon^{(2 / \alpha)-1} & \text { if } 0<\alpha<2, \\ \varepsilon^{2} & \text { if } \alpha=0 \\ \varepsilon^{2 /|\alpha|} & \text { if } \alpha<0 .\end{cases}
$$

To complete the proof of Theorem 4.2 it suffices to show that $\left\{c_{\varepsilon}\right\}$ is Cauchy as $\varepsilon \rightarrow 0$. Indeed, for $0<\varepsilon^{\prime}<\varepsilon$, by Lemmas 4.1 and 4.2 we have

$$
\begin{aligned}
c_{\varepsilon}-c_{\varepsilon^{\prime}} & \equiv \lim _{|x| \rightarrow 0} E^{P_{x}^{0}}\left[f\left(X^{0}\left(\tau_{0 \varepsilon}\right)\right)-f\left(X^{0}\left(\tau_{0 \varepsilon^{\prime}}\right)\right)\right] / q(x) \\
& =\lim _{|x| \rightarrow 0} E^{P_{x}^{0}}\left[\tau_{\varepsilon^{\prime}}<\tau_{0} ; \int_{\tau_{\varepsilon^{\prime}}}^{\tau_{0 \varepsilon}}\left\{k\left(X^{0}(t)\right)-\gamma f\left(X^{0}(t)\right)\right\} d t\right] / q(x) \\
& \leqslant C \limsup _{|x| \rightarrow 0} E^{P_{x}^{0}}\left[\tau_{0 \varepsilon}\right] / q(x), \quad \text { where } C \equiv \sup _{x \in R^{d}}\{(|k|+\gamma|f|)(x)\}<\infty \\
& =O(\beta(\varepsilon)) .
\end{aligned}
$$


Now $\beta(\varepsilon) \rightarrow 0$ as $\varepsilon \rightarrow 0$ and so $c_{\varepsilon} \rightarrow c$ for some constant $c$ as $\varepsilon \rightarrow 0$. Since all of the above limits and estimates have been uniform in $x /|x|$, Theorem 4.2 follows from letting $\varepsilon \rightarrow 0$ in (4.22).

The next theorem enables us to obtain estimates for $R_{\gamma}^{0} 1$ and $R_{\gamma}^{0} k$ when $k \in C_{b}\left(R^{d}\right)$ has compact support in $R^{d} \backslash\{0\}$. For this theorem, let $K_{\nu}, I_{\nu}$ denote the modified Bessel functions of order $\nu=|\alpha| / 2$ which satisfy

$$
w^{2} g^{\prime \prime}(w)+w g^{\prime}(w)-\left(w^{2}+\nu^{2}\right) g(w)=0 \text { for } w \in(0, \infty)
$$

with $g(w)=K_{\nu}(w)$ or $I_{\nu}(w)$. Further details on the properties of $K_{\nu}$ and $I_{\nu}$ used below can be found in Abramowitz and Stegun [1, pp. 374-378].

Theorem 4.3. Let $\lambda>0, \varepsilon>0$, and let $h$ be defined by (3.15). For $y \in[0, \infty)$, define

$$
g_{1}(y)= \begin{cases}\frac{\sqrt{y} I_{\nu}\left(\sqrt{2 \lambda \alpha^{2}} y^{1 / \alpha}\right)}{\sqrt{\varepsilon} I_{\nu}\left(\sqrt{2 \lambda \alpha^{2}} \varepsilon^{1 / \alpha}\right)} & \text { if } \alpha>0, \\ \frac{I_{0}(\sqrt{2 \lambda} y)}{I_{0}(\sqrt{2 \lambda} \varepsilon)} & \text { if } \alpha=0, \\ \frac{\sqrt{y} I_{\nu}\left(\sqrt{2 \lambda \alpha^{2}} y^{1 / \alpha}\right)}{\sqrt{\varepsilon^{-1}} I_{\nu}\left(\sqrt{2 \lambda \alpha^{2}} \varepsilon^{-1 / \alpha}\right)} & \text { if } \alpha<0, y \neq 0,\end{cases}
$$

and

$$
g_{2}(y)=c \sqrt{y} K_{\nu}\left(\sqrt{2 \lambda \alpha^{2}} y^{1 / \alpha}\right) \quad \text { if } \alpha>0
$$

where

$$
c \equiv 2^{1-(\alpha / 4)} \alpha^{\alpha / 2} \lambda^{\alpha / 4}(\Gamma(\alpha / 2))^{-1}
$$

is such that $g_{2}(0) \equiv \lim _{y \rightarrow 0} g_{2}(y)=1$ for $\alpha>0$.

For $\alpha \neq 0$ define $f_{1}=g_{1}(u)$, for $\alpha=0$ define $f_{1}=g_{1}\left(e^{u}\right)$, and for $\alpha>0$ define $f_{2}=g_{2}(u)$. Then for each $x \in R^{d} \backslash\{0\}$ such that $0<v(x) \leqslant \varepsilon$ we have

$$
E^{P_{x}^{0}}\left[\exp \left(-\lambda \int_{0}^{\tau_{0 \varepsilon}} h\left(X^{0}(s)\right) d s\right) ; \tau_{\varepsilon}<\tau_{0}\right]=f_{1}(x),
$$

and for each $x \in R^{d}$ and $\alpha>0$ we have

$$
E^{P_{x}^{0}}\left[\exp \left(-\lambda \int_{0}^{\tau_{0}} h\left(X^{0}(s)\right) d s\right)\right]=f_{2}(x) .
$$

Proof. It follows from (4.23) and the properties of $I_{\nu}, K_{\nu}$, including

$$
I_{\nu}(w)=\left(\frac{1}{2} w\right)^{\nu} / \Gamma(\nu+1)+o\left(w^{\nu}\right) \text { as } w \rightarrow 0,
$$

$K_{\nu}(w) \sim w^{-\nu}$ as $w \rightarrow 0$ for $\nu>0, K_{\nu}(w) \sim w^{-1 / 2} e^{-w}$ as $w \rightarrow \infty$, that for $\alpha>0$,

$$
\begin{aligned}
& \left\{\begin{array}{l}
\frac{1}{2} g_{1}^{\prime \prime}(y) y^{2-(2 / \alpha)}-\lambda g_{1}(y)=0 \quad \text { for } y \in(0, \infty), \\
g_{1}(0)=0, \quad g_{1}(\varepsilon)=1,
\end{array}\right. \\
& \left\{\begin{array}{l}
\frac{1}{2} g_{2}^{\prime \prime}(y) y^{2-(2 / \alpha)}-\lambda g_{2}(y)=0 \quad \text { for } y \in(0, \infty), \\
g_{2}(0)=1, \quad\left|g_{2}\right| \leqslant 1, \quad g_{2}(y) \rightarrow 0 \quad \text { as } y \rightarrow \infty ;
\end{array}\right.
\end{aligned}
$$


for $\alpha=0$,

$$
\left\{\begin{array}{l}
\frac{1}{2}\left\{g_{1}^{\prime \prime}(y)+y^{-1} g_{1}^{\prime}(y)\right\}-\lambda g_{1}(y)=0 \text { for } y \in(0, \infty) \\
g_{1}(\varepsilon)=1, \quad 0<g_{1}(y)<1 \text { for } 0 \leqslant y<\varepsilon
\end{array}\right.
$$

for $\alpha<0$,

$$
\left\{\begin{array}{l}
\frac{1}{2} g_{1}^{\prime \prime}(y) y^{2-(2 / \alpha)}-\lambda g_{1}(y)=0 \quad \text { for } y \in(0, \infty), \\
g_{1}\left(\varepsilon^{-1}\right)=1, \quad 0<\inf _{y \in\left[\varepsilon^{-1}, \infty\right)} g_{1}(y) \leqslant \sup _{y \in\left[\varepsilon^{-1}, \infty\right)} g_{1}(y)<\infty .
\end{array}\right.
$$

Then $f_{1}, f_{2}$ are functions in $C^{1}\left(R^{d} \backslash\{0\}\right) \cap \mathrm{W}_{\mathrm{loc}}^{2, p}\left(R^{d} \backslash\{0\}\right)$, where $p>d-1$ is as in $\S 3$. By a similar derivation to that of (3.23), using (4.26)-(4.29), we have on $R^{d} \backslash\{0\}$

$$
\begin{aligned}
L f_{1} & =\left\{\begin{array}{l}
\frac{1}{2} g_{1}^{\prime \prime}(u)|\nabla u|^{2} \quad \text { if } \alpha \neq 0, \\
\frac{1}{2}\left\{g_{1}^{\prime \prime}\left(e^{u}\right) e^{2 u}+g_{1}^{\prime}(u) e^{u}\right\}|\nabla u|^{2} \quad \text { if } \alpha=0
\end{array}\right. \\
& = \begin{cases}\frac{1}{2} g_{1}^{\prime \prime}(u) u^{2-(2 / \alpha)} h & \text { if } \alpha \neq 0, \\
\frac{1}{2}\left\{g_{1}^{\prime \prime}\left(e^{u}\right)+g_{1}^{\prime}(u) e^{-u}\right\} h & \text { if } \alpha=0\end{cases} \\
& =\lambda f_{1} h,
\end{aligned}
$$

and

$$
L f_{2}=\lambda f_{2} h \quad \text { if } \alpha>0 .
$$

Furthermore, $f_{1}$ is bounded on $\{x: 0<v(x) \leqslant \varepsilon\},\left.f_{1}\right|_{v^{-1}(\epsilon)}=1$, and if $\alpha>0$, $f_{1}(0)=0, f_{2}(0)=1, f_{2}(x) \rightarrow 0$ as $|x| \rightarrow \infty$; if $\alpha \leqslant 0$,

$$
0<\inf _{0<v(x) \leqslant \varepsilon} f_{1}(x) \leqslant \sup _{0<\nu(x) \leqslant \varepsilon} f_{1}(x)<\infty .
$$

Let $0<r<\varepsilon$ and $x \in\{z: r<v(z) \leqslant \varepsilon\}$. As in Lemma 3.1, the martingale property (2.1) with $\tau_{r \varepsilon}$ in place of $\tau_{0}$ extends to $f_{1}$ and $f_{2}$, so that by (4.30)-(4.31),

$$
\left\{M_{t}^{i}=f_{i}\left(X^{0}\left(t \wedge \tau_{r \varepsilon}\right)\right)-\int_{0}^{t \wedge \tau_{r \varepsilon}}\left(\lambda f_{i} h\right)\left(X^{0}(s)\right) d s, \mathscr{F}_{t \wedge \tau_{r \varepsilon}}^{0}, t \geqslant 0\right\}
$$

is a $P_{x}^{0}$-martingale for $i=1,2$.

Since

$$
V_{t} \equiv \exp \left(-\lambda \int_{0}^{t \wedge \tau_{r f}} h\left(X^{0}(s)\right) d s\right) \quad \text { and } \quad U_{t}^{i} \equiv \int_{0}^{t \wedge \tau_{r \varepsilon}}\left(\lambda f_{i} h\right)\left(X^{0}(s)\right) d s
$$

are locally of bounded variation, by the product rule of stochastic calculus we have, $P_{x}^{0}$-a.s.,

$$
d\left(V_{t}\left(M_{t}^{i}+U_{t}^{i}\right)\right)=V_{t} d M_{t}^{i}+V_{t} d U_{t}^{i}+\left(M_{t}^{i}+U_{t}^{i}\right) d V_{t} .
$$

The first term on the right of (4.32) integrates to a $P_{x}^{0}$-martingale since $M_{t}^{i}$ is such a martingale and $\left|V_{t}\right| \leqslant 1$. The last two terms in (4.32) sum to zero. Hence we may conclude that

$$
\left\{V_{t}\left(M_{t}^{i}+U_{t}^{i}\right) \equiv \exp \left(-\lambda \int_{0}^{t \wedge \tau_{r \varepsilon}} h\left(X^{0}(s)\right) d s\right) f_{i}\left(X^{0}\left(t \wedge \tau_{r \varepsilon}\right)\right), \mathscr{F}_{t \wedge \tau_{r \varepsilon}}^{0}, t \geqslant 0\right\}
$$


is a $P_{x}^{0}$-martingale for $i=1,2$. Then taking expectations for $i=1$ and letting $t \rightarrow \infty$ and then $r \rightarrow 0$, we obtain by bounded convergence (recall $\tau_{0 \varepsilon}<\infty P_{x}^{0}$-a.s.)

$$
E^{P_{x}^{0}}\left[\equiv \exp \left(-\lambda \int_{0}^{\tau_{0 \varepsilon}} h\left(X^{0}(s)\right) d s\right) f_{1}\left(X^{0}\left(\tau_{0 \varepsilon}\right)\right)\right]=f_{1}(x) .
$$

Note that for $0<v(x) \leqslant \varepsilon$, if $\alpha \leqslant 0, \tau_{0 \varepsilon}=\tau_{\varepsilon} P_{x}^{0}$-a.s., and if $\alpha>0, f_{1}(0)=0$, and for all $\alpha,\left.f_{1}\right|_{v^{-1}(\varepsilon)}=1$. Consequently, for any $\alpha, f_{1}\left(X^{0}\left(\tau_{0 \varepsilon}\right)\right)=1_{\left\{\tau_{\varepsilon}<\tau_{0}\right\}} P_{x}^{0}$-a.s. and (4.24) follows from (4.34).

When $\alpha>0$, by taking the expectation of the martingale (4.33) with $i=2$ and letting $t \rightarrow \infty$, then $r \rightarrow 0$, and then $\varepsilon \rightarrow \infty$, it follows, since $f_{2}$ is bounded and $\tau_{0}<\infty P_{x}^{0}$-a.s., that

$$
E^{P_{x}^{0}}\left[\exp \left(-\lambda \int_{0}^{\tau_{0}} h\left(X^{0}(s)\right) d s\right) f_{2}\left(X^{0}\left(\tau_{0}\right)\right)\right]=f_{2}(x)
$$

for all $x \in R^{d} \backslash\{0\}$. Since $f_{2}(0)=1,(4.25)$ follows immediately, even for $x=0$.

Proof OF THEOREM 4.1. Let $\gamma, \beta$ and $k$ satisfy the hypotheses of Theorem 4.1 and let $\varepsilon>0$ be so small that $\{x: 0 \leqslant v(x) \leqslant \varepsilon\}$ does not meet the support of $k$.

Consider $x \in R^{d} \backslash\{0\}$. First suppose $\alpha \leqslant 0$. Then, $P_{x}^{0}\left(\tau_{0}=\infty\right)=1$ and so by Theorem 4.2

$$
R_{\gamma}^{0} k(x) / R_{\beta}^{0} 1(x)=\beta R_{\gamma}^{0} k(x) \rightarrow \beta c(\gamma, k) \equiv c(\gamma, \beta, k)
$$

uniformly as $|x| \rightarrow 0$. If $0 \neq k \geqslant 0$, then by the choice of $\varepsilon$, for $0<v(x)<\varepsilon$,

$$
\begin{aligned}
R_{\gamma}^{0} k(x) & =E^{P_{x}^{0}}\left[e^{-\gamma \tau_{\varepsilon}} R_{\gamma}^{0} k\left(X^{0}\left(\tau_{\varepsilon}\right)\right)\right] \\
& \geqslant E^{P_{x}^{0}}\left[e^{-\gamma \tau_{\varepsilon}}\right]\left\|R_{\gamma}^{0} k\right\|_{\varepsilon}
\end{aligned}
$$

where $\left\|R_{\gamma}^{0} k\right\|_{\varepsilon}=\inf \left\{R_{\gamma}^{0} k(z): v(z)=\varepsilon\right\}>0$. Moreover, by Theorem 4.3, for $c_{1}$ as defined in (3.20),

$$
\begin{aligned}
E^{P_{x}^{0}}\left[e^{-\gamma \tau_{\varepsilon}}\right] & \geqslant E^{P_{x}^{0}}\left[\exp \left(-\gamma c_{1}^{-1} \int_{0}^{\tau_{0 \varepsilon}} h\left(X^{0}(s)\right) d s\right) ; \tau_{\varepsilon}<\tau_{0}\right] \\
& =f_{1}(x) \quad \text { with } \lambda=\gamma c_{1}^{-1} .
\end{aligned}
$$

Now as $|x| \rightarrow 0, u(x)^{1 / \alpha} \rightarrow 0$ if $\alpha<0$ and $e^{u(x)} \rightarrow 0$ if $\alpha=0$, and so

$$
\begin{aligned}
& f_{1}(x)=\left\{\begin{array}{l}
\frac{\sqrt{u(x)} I_{\nu}\left(\sqrt{2 \lambda \alpha^{2}} u(x)^{1 / \alpha}\right)}{\sqrt{\varepsilon^{-1}} I_{\nu}\left(\sqrt{2 \lambda \alpha^{2}} \varepsilon^{-1 / \alpha}\right)} \quad \text { if } \alpha<0, \\
\frac{I_{0}\left(\sqrt{2 \lambda} e^{u(x)}\right)}{I_{0}(\sqrt{2 \lambda} \varepsilon)} \quad \text { if } \alpha=0
\end{array}\right. \\
& \sim\left\{\begin{array}{l}
\sqrt{u(x)} u(x)^{\nu / \alpha}=1 \quad \text { if } \alpha<0, \\
I_{0}(0)>0 \text { if } \alpha=0 .
\end{array}\right.
\end{aligned}
$$

Here we have used the facts that $I_{\nu}(w) \sim w^{\nu}$ as $w \rightarrow 0$ and $\nu \equiv|\alpha| / 2=-\alpha / 2$ if $\alpha<0$. By combining (4.36)-(4.37), we conclude that if $0 \neq k \geqslant 0$,

$$
c(\gamma, k)=\lim _{|x| \rightarrow 0} R_{\gamma}^{0} k(x)>0,
$$

and hence so too is $c(\gamma, \beta, k) \equiv \beta c(\gamma, k)$. Thus, Theorem 4.1 holds if $\alpha \leqslant 0$. 
Now suppose $\alpha>0$. For $c_{2}$ as defined in (3.20), by Theorem 4.3 we have

$$
\begin{aligned}
R_{\beta}^{0} 1(x) & =\beta^{-1}\left(1-E^{P_{x}^{0}}\left[e^{-\beta \tau_{0}}\right]\right) \\
& \geqslant \beta^{-1}\left(1-E^{P_{x}^{0}}\left[\exp \left(-\beta c_{2}^{-1} \int_{0}^{\tau_{0}} h\left(X^{0}(s)\right) d s\right)\right]\right) \\
& =\beta^{-1}\left(1-f_{2}(x)\right) \quad \text { with } \lambda=\beta c_{2}^{-1} .
\end{aligned}
$$

By [1, 9.6.2 and 9.6.10], as $w \rightarrow 0$,

$$
w^{\nu} K_{\nu}(w)= \begin{cases}2^{\nu-1} \Gamma(\nu)-a_{\nu} w^{2 \wedge(2 \nu)}+o\left(w^{2 \wedge(2 \nu)}\right) & \text { if } \nu \neq 1, \\ 2^{\nu-1} \Gamma(\nu)+a_{\nu} w^{2} \ln w+O\left(w^{2}\right) & \text { if } \nu=1,\end{cases}
$$

where the $a_{\nu}$ are strictly positive constants.

It follows from this and the definition of $g_{2}$ that

$$
\begin{aligned}
1-f_{2}(x) & =1-g_{2}(u(x)) \\
& = \begin{cases}a_{\nu}^{\prime} u(x)^{(2 / \alpha) \wedge 1}+o\left(u(x)^{(2 / \alpha) \wedge 1}\right) & \text { if } \alpha \neq 2, \\
-a_{\nu}^{\prime} u(x)^{2 / \alpha} \ln u(x)^{1 / \alpha}+o\left(u(x)^{2 / \alpha}\right) & \text { if } \alpha=2,\end{cases}
\end{aligned}
$$

where the $a_{\nu}^{\prime}$ are strictly positive constants depending on $\lambda$ and $\varepsilon$ as well as $\nu=|\alpha| / 2$. Then, by dividing the above by $u(x)$, letting $|x| \rightarrow 0$, and combining the result with (4.38) we obtain

$$
\liminf _{|x| \rightarrow 0} \frac{R_{\beta}^{0} 1(x)}{u(x)} \geqslant \begin{cases}\beta^{-1} a_{\nu}^{\prime} & \text { if } 0<\alpha<2 \\ +\infty & \text { if } \alpha \geqslant 2 .\end{cases}
$$

By replacing $\geqslant$ and $c_{2}$, respectively, by $\leqslant$ and $c_{1}$ in the above argument, we conclude that there is a constant $a_{\nu}^{\prime \prime}>0$ such that

$$
\limsup _{|x| \rightarrow 0} \frac{R_{\beta}^{0} 1(x)}{u(x)} \leqslant \begin{cases}\beta^{-1} a_{\nu}^{\prime \prime} & \text { if } 0<\alpha<2, \\ +\infty & \text { if } \alpha \geqslant 2 .\end{cases}
$$

On the other hand, for $0<v(x)<\varepsilon$,

$$
\begin{aligned}
R_{\gamma}^{0} k(x) & =E^{P_{x}^{0}}\left[e^{-\gamma \tau_{0 \varepsilon}} R_{\gamma}^{0} k\left(X^{0}\left(\tau_{0 \varepsilon}\right)\right)\right] \\
& \leqslant E^{P_{x}^{0}}\left[\exp \left(-\gamma c_{2}^{-1} \int_{0}^{\tau_{0 \varepsilon}} h\left(X^{0}(s)\right) d s\right) ; \tau_{\varepsilon}<\tau_{0}\right]\|k\|_{\gamma, \infty} \\
& =f_{1}(x)\|k\|_{\gamma, \infty} \quad \text { with } \lambda=\gamma c_{2}^{-1},
\end{aligned}
$$

where $\|k\|_{\gamma, \infty}=\gamma^{-1} \sup _{z \in R^{d}}|k(z)|<\infty$. Now as $w \rightarrow 0$,

$$
I_{\nu}(w)=\left(\frac{1}{2} w\right)^{\nu} / \Gamma(\nu+1)+o\left(w^{\nu}\right)
$$

and consequently, as $|x| \rightarrow 0$,

$$
f_{1}(x)=g_{1}(u(x))=\frac{\sqrt{u(x)} I_{\nu}\left(\sqrt{2 \lambda \alpha^{2}} u(x)^{1 / \alpha}\right)}{\sqrt{\varepsilon} I_{\varepsilon}\left(\sqrt{2 \lambda \alpha^{2}} \varepsilon^{1 / \alpha}\right)}=b_{\nu} u(x)+O(u(x)),
$$

where $b_{\nu}>0$ is a constant depending on $\nu, \varepsilon$ and $\lambda=\gamma c_{2}^{-1}$. By combining this with (4.41) we obtain

$$
\limsup _{|x| \rightarrow 0} \frac{R_{\gamma}^{0} k(x)}{u(x)} \leqslant b_{\nu}\|k\|_{\gamma, \infty}
$$


By replacing $\leqslant, \quad c_{2}$ and $\|k\|_{\gamma, \infty}$, respectively, by $\geqslant, \quad c_{1}$ and $\left\|R_{\gamma}^{0} k\right\|_{\varepsilon}=$ $\inf _{z \in v^{-1}(\varepsilon)} R_{\gamma}^{0} k(z)$, from (4.41) onwards, we conclude that, for $0 \neq k \geqslant 0$,

$$
\liminf _{|x| \rightarrow 0} \frac{R_{\gamma}^{0} k(x)}{u(x)} \geqslant b_{\nu}^{\prime}\left\|R_{\gamma}^{0} k\right\|_{\varepsilon}>0
$$

for some constant $b_{\nu}^{\prime}>0$ depending on $\nu, \varepsilon, \gamma c_{1}^{-1}$. Note that the limits in (4.39)-(4.40), (4.42)-(4.43) are uniform in $x /|x|$.

It follows from (4.39)-(4.40) that, for $0<\alpha<2$, the constant $c(\beta, 1)$ defined via Theorem 4.2 satisfies $0<c(\beta, 1)<\infty$. Consequently, for $0<\alpha<2$,

$$
\frac{R_{\gamma}^{0} k(x)}{R_{\beta}^{0} 1(x)}=\frac{R_{\gamma}^{0} k(x) / u(x)}{R_{\beta}^{0} 1(x) / u(x)} \rightarrow \frac{c(\gamma, k)}{c(\beta, 1)} \equiv c(\gamma, \beta, k)
$$

uniformly as $|x| \rightarrow 0$, where $c(\gamma, \beta, k)<\infty$, and if $0 \not \equiv k \geqslant 0$, it follows from (4.43) that $c(\gamma, k)>0$ and hence $c(\gamma, \beta, k)>0$. Thus Theorem 4.1 holds if $0<\alpha<2$.

Finally, if $\alpha \geqslant 2$, by (4.39) and (4.42)

$$
\limsup _{|x| \rightarrow 0} \frac{\left|R_{\gamma}^{0} k(x)\right|}{R_{\beta}^{0} 1(x)} \leqslant \limsup _{|x| \rightarrow 0} \frac{R_{\gamma}^{0}|k|(x) / u(x)}{R_{\beta}^{0} 1(x) / u(x)} \leqslant \frac{b_{\nu}\|k\|_{\gamma, \infty}}{\liminf _{|x| \rightarrow 0} R_{\beta}^{0} 1(x) / u(x)}=0,
$$

uniformly in $x /|x|$.

5. Continuation from the pole. It follows from Theorem 4.1 and the work of Rogers [11], particularly the discussion of the Martin compactification on pp. 249-251 of [11], that there is a single Martin boundary point for $X^{0}$ at the origin so that the Martin kernel $K(x, y)$ defined for $(x, y) \in\left(R^{d} \backslash\{0\}\right) \times\left(R^{d} \backslash\{0\}\right)$ where

$$
\int_{R^{d} \backslash\{0\}} K(x, y) k(y) d y=R_{\gamma}^{0} k(x) / R_{\gamma}^{0} 1(x)
$$

for all $k \in C_{c}\left(R^{d} \backslash\{0\}\right)$, can be extended continuously to $x=0$ :

$$
\int_{R^{d} \backslash\{0\}} K(0, y) k(y) d y=\lim _{x \rightarrow 0} R_{\gamma}^{0} k(x) / R_{\gamma}^{0} 1(x)= \begin{cases}c(\gamma, \gamma, k) & \text { if } \alpha<2, \\ 0 & \text { if } \alpha \geqslant 2 .\end{cases}
$$

It further follows that, for $\alpha<2$, there is a unique strong Markov process with continuous paths in $R^{d}$ that behaves like $X^{0}$ away from the origin, but the amount of time that it spends at the origin has Lebesgue measure zero. Indeed, for $\gamma>0$ and $k \in C_{c}\left(R^{d}\right)$ with compact support in $R^{d} \backslash\{0\}$, the resolvent of $X$ is given by (cf. [11, (2), (33)])

$$
R_{\gamma} k(x) \equiv E^{P_{x}}\left[\int_{0}^{\infty} e^{-\gamma t} k(X(t)) d t\right]=R_{\gamma}^{0} k(x)+E^{P_{x}^{0}}\left[e^{-\gamma \tau_{0}}\right] \gamma^{-1} c(\gamma, \gamma, k) .
$$

Conversely, if $\alpha \geqslant 2, K(0, y)=0$ for all $y \in R^{d} \backslash\{0\}$ and it follows from [11] that there is no such process $X$ in this case.

Following the approach in Varadhan and Williams [15], for $\alpha<2, X$ can be characterized in law as follows using families of martingales. We can use martingales here, rather than submartingales as in [15], because there are no boundary points besides the origin. The process $X$ is uniquely characterized in law as the process with continuous sample paths in $R^{d}$ and associated probability measures $\left\{P_{x}, x \in R^{d}\right\}$ 
(one for each starting state $x$ ) satisfying the following three properties:

(i) $P_{x}(X(0)=x)=1$.

(ii) For each $f \in C_{c}^{2}\left(R^{d}\right)$ with compact support in $R^{d} \backslash\{0\}$,

$$
\left\{f(X(t))-\int_{0}^{t} L f(X(s)) d s, \mathscr{F}_{t}, t \geqslant 0\right\}
$$

is a $P_{x}$-martingale, where $\mathscr{F}_{t} \equiv \sigma\{X(s): 0 \leqslant s \leqslant t\}$.

(iii)

$$
E^{P_{x}}\left[\int_{0}^{\infty} 1_{\{0\}}(X(t)) d t\right]=0
$$

One question that remains unanswered here and provides an area for further research is the following: Is there a simple criterion, expressed in terms of $\mu$ and $d$, for determining whether $\alpha \geqslant 2$ ? When $\mu \equiv$ constant, the answer is known: $\alpha=2-$ $d-\mu$ and so $\alpha \geqslant 2$ is equivalent to $\mu \leqslant-d$.

Acknowledgment. The author would like to thank J. W. Pitman for posing questions which led to the study of the process $X^{0}$ and for the reference to the paper by Kendall [7]. For several discussions during the progress of this work, thanks are also expressed to S. R. S. Varadhan and P. March of the Courant Institute.

\section{REFERENCES}

1. M. Abramowitz, and I. A. Stegun, Handbook of mathematical functions, National Bureau of Standards, Appl. Math. Series 55, 1968.

2. K. L. Chung, Lectures from Markov processes to Brownian motion, Springer, New York, 1982.

3. M. G. Crandall and P. H. Rabinowitz, Bifurcation, perturbation of simple eigenvalues and linearized stability, Arch. Rational Mech. Anal. 52 (1973), 161-180.

4. B. E. J. Dahlberg, Estimates of harmonic measure, Arch. Rational Mech. Anal. 65 (1977), 275-288.

5. D. Gilbarg and N. S. Trudinger, Elliptic partial differential equations of second order, Springer, New York, 1983.

6. K. Itô and H. P. McKean, Jr., Diffusion processes and their sample paths, Springer, New York, 1974.

7. D. G. Kendall, Pole-seeking Brownian motion and bird navigation, J. Roy. Statist. Soc. Ser. B 36 (1974), 365-417.

8. M. G. Krein, and M. A. Rutman, Linear operators leaving invariant a cone in a Banach space, Amer. Math. Soc. Transl. (1) 10 (1962), 199-325.

9. R. S. Lipster and A. N. Shiryayev, Statistics of random processes, I, General theory, Springer, New York, 1977.

10. D. Revuz, Markov chains, 2nd ed., North-Holland, Amsterdam, 1984.

11. L. C. G. Rogers, Itô excursion theory via resolvents, Z. Wahrsch. Verw. Gcbiete 63 (1983), 237-255.

12. D. W. Stroock and S. R. S. Varadhan, Diffusion processes with continuous coefficients, Comm. Pure Appl. Math. 22 (1969), I: 345-400, II: 479-530.

13. _ Diffusion processes with boundary conditions, Comm. Pure Appl. Math. 24 (1971), 147-225.

14. __ Multidimensional diffusion processes, Springer, New York, 1979.

15. S. R. S. Varadhan, and R. J. Williams, Brownian motion in a wedge with oblique reflection, Comm. Pure Appl. Math. 38 (1985), 405-443.

16. R. J. Williams, Recurrence classification and invariant measure for reflected Brownian motion in a wedge, Ann. Probab. (to appear).

Department of Mathematics, University of California, La Jolla, California 92093 